\title{
Flame ignition in the counterflow configuration: Reassessing the experimental assumptions
}

\author{
Abtin Ansari, Fokion N. Egolfopoulos \\ Department of Aerospace and Mechanical Engineering \\ University of Southern California, Los Angeles, California 90089-1453, USA
}

Full length article accepted for publication in Combustion and Flame

Running title: Flame ignition in the counterflow configuration

\section{Corresponding Author:}

Fokion N. Egolfopoulos

Department of Aerospace and Mechanical Engineering, University of Southern California

Los Angeles, California 90089-1453, USA

Phone: 213-740-0480

Fax: 213-740-8071

Email: egolfopo@usc.edu

September 14, 2016 


\title{
Flame ignition in the counterflow configuration: Reassessing the experimental assumptions
}

\author{
Abtin Ansari, Fokion N. Egolfopoulos \\ Department of Aerospace and Mechanical Engineering, \\ University of Southern California, Los Angeles, California 90089-1453, USA
}

\begin{abstract}
The counterflow configuration is widely used to study experimentally premixed and non-premixed flame ignition, with the advantage being that the data can be modeled using quasi one-dimensional codes. In this study, experiments and direct numerical simulations were carried out in order to assess the validity of the assumptions of the one-dimensional formulation. Experimentally, particle image velocimetry, shadowgraph, and a high-speed camera were employed to characterize the flow field before ignition, and to capture the ignition position and further evolution of the flame. The modeling involved axisymmetric numerical simulations using detailed molecular transport and chemical kinetic models. Both experiments and simulations revealed that if solid surfaces are present in the vicinity of the jets exit, the flow separates generating recirculation zones that are unstable and result in the bifurcation of the flow field. As a result, for a given set of boundary conditions at the burners' exits, there exists two possible stable states of the flow field which have different velocity and scalars distribution, and the fuel concentration at which ignition occurs was determined to differ for these two states. A novel approach is proposed to correct for the unavoidable radial non-uniformity of the temperature profile at the exit of the heated jet and the conditions that do not result in bifurcation are outlined, so that the results from one-dimensional codes can be compared to the data with confidence.
\end{abstract}

Keywords: Counterflow flames, laminar flames, flame ignition, direct numerical simulations, bifurcation 


\section{Introduction}

The counterflow configuration is in principle a well-controlled environment in which global flame properties such as ignition temperatures, laminar flame speeds, and extinction strain rates can be measured and used as validation targets for kinetic models under the assumption that the flow-field is (quasi) one-dimensional (1-D). If the axial velocity and scalars exhibit zero radial gradients along the axis of symmetry, then a similarity solution exist and as a result, only the axis of symmetry needs to be modeled [1] reducing thus the computational cost and removing ambiguities associated with boundary conditions (BC) that need to be characterized in multi-dimensional configurations (e.g., [2]).

The validity of the 1-D assumption has been assessed in several studies both in the counterflow configuration (e.g., [3-11]) and stagnation flows (e.g., [12-17]) for conditions that could be encountered in experiments. Chelliah et al. [3] demonstrated that the requirement of zero radial gradient of the axial velocity in the counterflow configuration can be relaxed by implementing the measured value of this quantity as a BC. It has been shown that parabolic burner exit velocity profiles could result in notable radial non-uniformities of velocities and scalars in the flow-field [4,5,7]. For non-premixed flames, it has been shown [8] that for an Mshape burner exit velocity profile, the stagnation plane and the stoichiometric surface exhibit notable curvature at large radii. As a result, heat release, scalar dissipation rates, and temperature peak away from the centerline, which apparently violates the 1-D assumptions. Mittal et al. [9] demonstrated that a non-uniform M-shape burner exit velocity profile, caused by a dip in the centerline, induces a temperature curvature that has a significant effect on laminar flame speeds obtained through extrapolations.

Niemann et al. [10] demonstrated that careful design of large diameter straight tube burners with flow straightening screens, can produce velocity profiles in counterflowing non-reacting cold air jets, which satisfy the 1-D assumptions within an accuracy of 5\%. Johnson et al. [11] showed computationally that the burnerdesign proposed by Niemann et al. [10] exhibit negligible two-dimensionality for non-premixed $\mathrm{H}_{2}$ flames by thoroughly comparing the terms in momentum and energy equations generated by 1-D and 2-D approaches. The required design considerations for appropriately reproducing one-dimensionality using contoured-nozzles have also been identified in the same study [11].

Bergthorson et al. [12] reported that 1-D formulation assumptions would be guaranteed in premixed flames in the stagnation flow configuration for properly designed contoured-nozzles [13] if the measured velocity BC's at suitably chosen point are implemented in the simulations. Success of 1-D formulation in such designs was further verified by comparing to the measured velocity, temperature and NO concentration profiles $[13,17]$. Furthermore, Bergthorson et al. [16] verified the validity of the 1-D formulation in non-reacting stagnation 
flows by applying the approach of Chelliah et al [3]. On the other hand, Bouvet et al. [15] reported that due to variations of pressure curvature along the centerline, 1-D models could not reproduce the measured velocity profiles in stagnation flames. However, the conclusions for the non-reacting cases were flawed since the 1-D model was used outside its domain of applicability [11,16,18-20].

While the aforementioned studies on potential multi-dimensional effects are meritorious, they have been carried out in the presence of a flame with some exceptions $([7,10,16])$. On the other hand, when the counterflow configuration is used to study flame ignition, the physical and chemical processes that lead to ignition evolve in the absence of a flame and are controlled by fluid mechanics, heat and mass transfer to and from the ignition kernel whose temperature is modest compared to a flame, and the relatively slow evolution of the radical pools. Additionally, the presence of one heated jet could add to the complexity of the flow field. Studies of multi-dimensional effects on the ignition of counterflow flames have yet to appear. Furthermore, it is generally accepted that stretch dampens any disturbances resulting thus in a steady flow field (e.g., [10]).

However, what may be less known is that while the stagnation flow is a stable configuration [21], the counterflow configuration in the absence of a flame, is in principle hydrodynamically unstable resulting in two stable states for the same BC's (e.g., [22-25]). More specifically, the symmetry of the flow field could break, and for identical flow BC's, the stagnation plane could be established at two different locations away from the symmetry plane between the nozzles. This asymmetric behavior is observed when a critical Reynolds number based on nozzle diameter $\left(\operatorname{Re}_{\text {crit }}\right)$ is reached (e.g., [23,25]). More specifically, experiments and simulations suggest that even when the momenta of two opposing jets are equal, the resulting flow field may be asymmetric and exhibit multiple steady states. The transition from a single steady state to "bi-stability" is an indication of "pitchfork bifurcation" and has been introduced in the analysis of dynamical systems (e.g., [26]); this is a manifestation of the inherent non-linearity of the Navier-Stokes equations. Figure 1 depicts the schematic of a typical stability diagram for counterflow jets indicating that beyond the $R \mathrm{e}_{\text {crit }}$ value the single stable solution bifurcates (e.g., [23,25]).

In bifurcation studies, the state at which the stagnation point is closer to the lower burner is denoted as the "lower branch" while the other state is called the "upper branch" (e.g., [23,25]). Since 1-D codes simulate only the symmetric branch, after the $R \mathrm{e}_{\text {crit }}$ value has been reached, the fidelity of computed results based on 1-D assumptions must be assessed.

Physically, this bifurcation is partially attributed to the Coanda effect, which is the tendency of the flow stream to attach to adjacent surfaces (e.g., [27]). At high enough velocities, the flow exiting the nozzles can eventually separate and create recirculation zone(s) next to any solid surface. These recirculation zones create 
radial velocity profiles that satisfy the necessary condition to develop local instability according to Rayleigh and Fjortoft criterion as shown in classical shear flow studies (e.g., [28,29]).

On the other hand, Rolon et al. [22] showed experimentally that for counterflowing ambient-temperature air jets, the stagnation plane is not located in the middle plane between the nozzles even when all surfaces surrounding the nozzle exits and resulting recirculation zones are removed. It was further shown, that the offset of the stagnation plane location increases with the nozzle separation distance $(L)$.

In counterflow flame experiments, flow-surface interactions are frequently unavoidable. First, the two impinging jets leave the nozzles that have rims with a finite thickness. Second, cooling jackets and coils are used to protect the nozzles from thermal stresses (e.g., [8,30]), and insulation jackets are an essential part of the counterflow ignition experiments in which high temperatures need to be reached at the exit of one nozzle (e.g., [31]). Finally, the experiments are performed typically in closed chambers in order to minimize ambient disturbances, establish inert environment for safety reasons, and reach pressures of relevance to applications (e.g., [32-34]).

Ciani et al. [25] reported a significant hysteresis between the two stable solutions in counterflowing air jets. It was shown that once the flow establishes itself at one state, it could not be switched to the other unless the momentum at the exit of the nozzle that is closer to the stagnation plane increases by a certain amount. The excess amount of momentum required for the hysteretic transition was found to increase with $R e$.

Bifurcation of non-isothermal counterflowing jets has been reported also. Conditions that result in steady [35] and unsteady behavior of the flow-field have been identified [24,36,37]. Those conditions were shown to depend on the velocity and temperature at the nozzle exit as well as $L$.

Stability analysis of counterflowing non-reacting jets has been performed for a wide range of $L$ values. The appropriate geometric parameter commonly used is the aspect ratio $\alpha \equiv L / D$, where $D$ is the jet diameter. Nevertheless, the range where flame studies are being performed is $0.3<\alpha<2$, i.e. the "free-floating" regime (e.g., [18-20]). In this regime, only stable and bi-stable states have been reported in axisymmetric configuration when the top jet has lower density, i.e. stable stratification [8,9,23-25,31-37].

Finally, it should be noted that in the presence of a flame, the heat release is intense resulting in high temperatures and thus notable buoyancy-driven convection at large radii, which has been shown to suppress the tendency of the flow-field to bifurcate [37]. This could be the reason that in the combustion literature, the stability of counterflowing jets has attracted less attention despite its wide use. To the authors' knowledge, the only bi-stability of reacting flows in counterflow configuration was reported by Ciani et al. [38] who observed a 
transition from a planar diffusion flame to an edge flame during extinction where the behavior was attributed to flow field exchange of stability from one steady state to another.

Based on these considerations and the possible existence of bi-stable states in counterflow flame ignition experiments in which a flame is not present, the main goal of this work was to investigate experimentally and computationally multi-dimensional effects and assess the validity of the 1-D model. Specifically, the present investigation aims to quantify the uncertainty that is introduced by bifurcation, thermal boundary layers, and actual experimental geometry and boundary conditions. It should be noted that such effects on the flow field have not been addressed adequately in previous studies, which have been largely carried out in the absence of temperature gradients that are unavoidable in counterflow flame ignition experiments. 


\section{Experimental approach}

\subsection{Method and apparatus}

Experiments were carried out under atmospheric pressure in a counterflow burner configuration shown schematically in Fig. 2. The apparatus consists of a straight upper quartz burner, from which a high temperature jet is directed downward against an ambient temperature $\mathrm{H}_{2} / \mathrm{N}_{2}$ jet that exits from the lower straight burner; for both burners $D=14.5 \mathrm{~mm}$. Air was used as the hot oxidizer to study the ignition of non-premixed $\mathrm{H}_{2}$ flames. Coflowing $\mathrm{N}_{2}$ streams with an annulus gap of $3 \mathrm{~mm}$ surround both jets. All gaseous flow rates were metered using sonic nozzles by controlling the pressure upstream of the orifice.

The design of the upper burner that heats the air differs from that used in previous studies [31-34,39-41]. More specifically, only external resistance heating wire (Kanthal A1) was used, and in order to obtain temperatures at the center of upper burner exit, $T_{\text {air }}$, up to $1400 \mathrm{~K}$, the length of the upper nozzle had to be chosen significantly larger than conventional quartz burners that use internal heating elements. The burner was an $85 \mathrm{~cm}$ long tube of which two segments were heated, each being $37 \mathrm{~cm}$ long. The power supplied to each heated segment was controlled separately using variable AC transformers. Two bare B-type thermocouples (Omega P30 R-008) were mounted on the outside surface of the tube at the end of each heated segment to control the temperature of the segment manually. The entire upper burner assembly was wrapped with an alumina silica blanket to minimize heat loss.

Temperature measurements were carried out using an R-type thermocouple (Omega P13 R-002) with a bead diameter of $120 \pm 25 \mu \mathrm{m}$ determined by electron microscopy. Details of the thermocouple assembly and measurements are elaborated in Appendix C of the Supplementary Material (SPM).

The lower burner was made of stainless steel mounted onto a multistage translational stand capable of translating about multiple axes in order to obtain desired alignment and separation distance between the burners. $L=D=14.5 \mathrm{~cm}$ was chosen for all experiments $(\alpha=1)$. A cooling coil was wrapped around the lower burner to prevent any temperature rise due to radiation emitted from the heated quartz burner and assure that the $\mathrm{H}_{2} / \mathrm{N}_{2}$ jet is maintained uniformly at an unburned temperature $T_{\mathrm{u}}=298 \mathrm{~K}$. Two fine-wire screens (200 mesh) were placed in the lower burner as flow straighteners; one $8 D$ and the other $10 D$ upstream of the burner exit. As a result, the lower burner exit velocity profile is of top-hat shape. The measured burner exit profiles are shown in Appendix B of SPM.

Another major difference between the current design and the previous generation of burners is the fact that no flow straightener was used in the upper nozzle. The burner is long enough for the flow field to become fully 
developed both thermally and hydrodynamically at its exit. Note that temperature and axial velocity profiles at the heated burner exit were reported to be parabolic when heated even when flow straighteners are used $[33,40,41]$. This is largely due to the higher thermal diffusivity and kinematic viscosity at high temperatures that result in thicker boundary layers. Given the non-uniformities at the burner exits, balancing the momenta between the two jets is involved and the details of the process followed in this study can be found in Appendix

\section{A of SPM}

Not using flow straighteners in the heated burner, several problems are eliminated. These include: (1) agglomeration of seeding particles in the colder mesh caused by thermophoresis; (2) loss of flow alignment due to thermal expansion and subsequent displacement of the mesh; (3) mechanical failure of the quartz tube due to thermal stresses caused by difference between the thermal expansion coefficients at the steel-quartz junction/interface; and (4) uncertainties caused by accounting for radiation correction performed on the measured temperature due to glowing mesh at high temperatures.

The system was allowed to reach a steady temperature profile before each experiment. Upon establishing the appropriate flow rates and temperatures at the two burner exits, the fuel flow rate was gradually increased until ignition was observed (e.g., [31]). This approach was preferred over raising $T_{\text {air }}$ until ignition happens, as the uncertainty in the fuel side flow rate is notably lower compared to that of $T_{\text {air }}$ (e.g., $\left.[33,41]\right)$. Moreover, if the supplied power to the heating wire is changed in an attempt to raise $T_{\text {air }}$, it was observed that the whole system has to be allowed to reach a new thermal equilibrium which can take up to twenty minutes due to the high heat capacity of the heated burner.

The measured $T_{\text {air }}$ at the state of ignition was defined as the ignition temperature ( $\left.T_{\text {ign }}\right)$ (e.g., [31]), once corrected for radiative/convective heat transfer to/from the bead [33]. The uncertainty of the final corrected temperature was determined to be less than $\pm 35 \mathrm{~K}$ at the maximum achievable $T_{\text {air }}$ incorporating the methodology of Brady et al. [41]; the uncertainty was determined to be mostly due to the uncertainty of thermocouple bead diameter measurements and its effect in radiation correction.

\subsection{Flow and flame visualization}

The flow was seeded with $\mathrm{Al}_{2} \mathrm{O}_{3}$ particles with a nominal diameter of $3 \mu \mathrm{m}$, and particle image velocimetry (PIV) was used to measure the 2-D flow field on a plane that passes through the system centerline. The PIV details can be found in previous studies by the authors (e.g., [30,43]). The axial flow velocities along the stagnation streamline and the local strain rate, $K$, werw derived based on the maximum absolute value of the 
axial velocity gradient on the fuel-side [31]. The uncertainty of the PIV measurements were determined by the methodology of Sarnacki et al. [20] and was found to be of the order of $\pm 0.04 \mathrm{~m} / \mathrm{s}$ while the $95 \%$ confidence limit of $K$ is \pm 30 s. $^{-1}$

High-speed recording of chemiluminescence from the flame along with shadowgraph imaging were performed as well, in order to further evaluate the qualitative flow conditions during ignition and the subsequent edge flame propagation (e.g., [44]). A direct Shadowgraph ensemble has been set up to visualize the stagnation plane and the flame as shown in Fig. 3. Light was gathered from $13 \mathrm{~mW}$ epoxy encased white LED and focused on a pinhole to generate a point source light. The diameter of the pinhole/aperture is adjustable and can be optimized to obtain the best illumination. Light rays were further parallelized inside the test section using a series of spherical lenses with a diameter of 2 inches and focused again at the camera lens after passing through the established flow field. A high-speed charge-coupled device (CCD) camera (X-Sream, XS-3) was placed very close to the focal point of the last lens. A frame rate of $500 \mathrm{~Hz}$ with the resolution of $1258 \times 1280$ pixels and an exposure time of $2 \mathrm{~ms}$ was utilized to obtain the best balance between contrast and resolution. This method was particularly efficient in determining $L$ and aligning the burners with a spatial resolution better than $0.15 \mathrm{~mm}$.

Flame luminosity was recorded to monitor the ignition location via the CCD camera with the same frame rate and exposure of shadowgraph experiments. The fuel stream was doped with a trace amount of methane to make the flame visible and with negligible effect on the reactivity of the system (e.g., [33,45]). Experiments were repeated without methane, and ignition was confirmed at the same conditions using shadowgraph. In order to verify that the ignition is taking place at the centerline, ignition had to be recorded at two different angles. Therefore, a flat mirror was utilized to reflect the ignition event at an angle with respect to the original position of the camera as shown in Fig. 3. Only after shots were taken from these two views multiple times, the actual position of ignition could be found.

$T_{\text {ign }}$ was first determined for each experiment, following which the flow field was established at fuel flow rates 3\% below the ignition point at $T_{\text {ign }}$ where recording commenced. Only after triggering the camera, the fuel flow rate was increased to the desired value for ignition. Five records were taken at the ignition point to assure repeatability and quantify the uncertainty of the ignition position $( \pm 3 \mathrm{~mm})$.

It should be noted that PIV measurements, shadowgraph and flame visualization were performed independently. The optical system for PIV measurements was mounted such that the laser light sheet was perpendicular to the light arrays of shadowgraph. 


\section{Modeling approach}

Transient numerical simulations of the 2-D axisymmetric problem involving coupled fluid dynamics, detailed chemistry, and transport are carried out using the laminarSMOKE code [46], which is an open-source computational package built on the OpenFOAM [47,48] platform and details are given in Appendix B of SPM. The laminarSMOKE code has been specifically validated for counterflow flames [49] and also used to simulate other laminar combustion phenomena in axisymmetric configuration such as low-pressure flame sampling [50]. Numerical simulations were performed for atmospheric non-premixed $\mathrm{H}_{2}$ flames at pre-ignition state with a $\mathrm{H}_{2}$ mole fraction $X_{\mathrm{F}}=0.2$ in the fuel jet. The $\mathrm{H}_{2} / \mathrm{O}_{2}$ sub-model of USC Mech-II [51] was utilized to describe the kinetics. First, (quasi) 1-D simulations were performed to estimate $T_{\text {ign }}$ for given $X_{\mathrm{F}}$ and $K$ using an opposed-jet code [1,52-54], and the maximum $T_{\text {air }}$ for the 2-D simulations was kept $20 \mathrm{~K}$ lower than the estimated $T_{\text {ign }}$ to make sure that ignition would not occur during the transient simulations. This is a safe assumption since the conditions chosen correspond to the second explosion limit of $\mathrm{H}_{2}$ where $T_{\text {ign }}$ is not sensitive to $K$ [33].

In order to investigate the effects of the burner-flow-walls interactions, three axisymmetric geometries were studied as depicted in Fig. 4. Geometry (a) corresponds to the experimental configuration used in this study and involves realistic velocity and temperature BC's, which are parabolic for the heated burner and nearly top-hat for the lower burner as shown in Appendix B of SPM. Since parabolic jet exit profiles in geometry (a) introduce undesirable 2-D complexities [4,5,7], it might be meaningless to compare the 1-D and 2-D results. Hence, the simulations were repeated for geometry (b), which have BC's with minimum radial non-uniformities complying with the assumptions of 1-D formulation [1,10]. Solid surfaces adjacent to coflow exits i.e., the insulation wrap for upper burner and the cooling jacket for lower burner as shown in Fig. 2 are denoted as disk walls in Fig. 4a. Since it has been reported that recirculation zones could be eliminated by removing disk walls and solid surfaces next to the jets exits [22], the simulations were repeated for geometry (c) with the ideal BC's of geometry (b) but with replacing disk walls with open boundaries. Performing 2-D simulations for this geometry is particularly useful since the mechanism leading to bifurcation is widely attributed to the recirculation zones $[22,23,25,28]$.

Table 1 summarizes the simulations performed and their correspondence and characteristics. Additionally, BC's, details of the various geometries, and detailed solution procedures are provided in Appendix B of SPM. 
To obtain both possible solution branches numerically in the presence of hysteresis an approach similar to Ciani et al. [25] was incorporated; an initial non-reacting flow field was established in a coarse grid at low enough $R e$ at which only one stable state exist. At this state, the inlet velocities and temperatures were fixed for the final desirable state but the kinematic viscosity in the momentum equation was artificially increased to achieve a low $R e$ solution. It was realized that the solution was already asymmetric even at $R e$ as low as 20 , which is thought to be a result of profound influence of buoyancy at low $R e$ [8]. Then, $R e$ was artificially increased in small steps by reducing the effective kinematic viscosity. The existence of the other stable branch was subsequently tested as follows. If the original axisymmetric solution was a lower (upper) branch then the flow field was perturbed by increasing (decreasing) the average velocity of the fuel side to twice (half) of the original value and simulation was continued until the flow establishes a new equilibrium. The new state can be categorized into one of the branches by observing the separation bubble sizes at the upper and lower disk walls at exit of the jets; the bubble at the lower disk wall for lower branch is always smaller than the corresponding one at upper wall (e.g., [25]). The opposite is true for the upper branch. Subsequently, the fuel side velocity was adjusted to the value at which the momenta of the two jets are balanced. If the transition was observed for the switched state, extra care had to be taken to assure the solution would not jump to the initial branch. $R e$ was then increased further and the procedure was repeated until both stable solutions were obtained. Having the two solutions, $R e$ can be further increased to reach to the final state where refinement procedure is performed. After refinement, the chemistry step solver was activated and the solution was tracked until steady state was reached for all species in the domain. 


\section{Results and discussion}

\subsection{Bifurcation effects}

All experiments were carried out for fixed $T_{\text {air }}=926 \mathrm{~K}$ and $K=360 \mathrm{~s}^{-1}$ and the ignition state was characterized by the $X_{\mathrm{F}}$ value that would lead to ignition. Ignition was found to occur consistently at two different $X_{\mathrm{F}}$ values namely $X_{\mathrm{F}}=0.18$ and $X_{\mathrm{F}}=0.22$, and the number of ignition occurrences at those two $X_{\mathrm{F}}$ values was found be approximately the same for more than 20 trials. The uncertainty of $X_{\mathrm{F}}$ was estimated using the methodology of Brady et al. [41] and it was determined to be less than 0.01. Therefore the observed differences of the $X_{\mathrm{F}}$ values at ignition cannot be attributed to experimental uncertainty.

Shadowgraph images were taken at $X_{\mathrm{F}}=0.15$, which corresponds to a pre-ignition state. For the same BC's, two stable states were found as shown in Fig. 5. The state of the flow-field was determined to be susceptible to external disturbances. For example, inserting the thermocouple may cause the transition between the two states. Also after extinguishing an ignited flame by turning off the fuel supply, the flow could be in a state different from that before ignition. This is a further proof that the flow does indeed switch between the aforementioned states. In the absence of the external disturbances, the two states are obtainable by modifying the cold jet flow rate similar to the numerical procedure outlined in Section 3.

The stagnation plane is expected to have a similar shape as the non-planar discontinuity shapes in the light intensity shown in Fig. 5 and the stagnation point along the centerline is closer the lower burner for the conditions of Fig. 5a. Therefore, the images shown in Figs. 5a and 5b correspond to the lower and upper branches respectively; recall that fuel (air) is supplied by the lower (upper) burner.

For both the upper and lower branches, axial velocity profiles along the centerline were measured and computed using 2-D simulations for geometry (a). The results are shown in Fig. 6 along with results from 1-D simulations. The coordinate system was chosen to be at the center of lower burner exit and the spatial distances were scaled by $D$, as outlined in Fig. 4. 2-D and original 1-D simulation results match at the nozzles exits for both branches. However, the position of the stagnation plane and the adjacent velocity field differ notably between the two formulations, such discrepancy might lead to misleading conclusions if the experimental results are compared to the 1-D simulations. It has been reported that the 1-D approach can capture the velocity profiles along the centerline if BC's are measured at the burner exits and implemented in the simulations (e.g., [3,16,17,20,52]). Although, this procedure was implemented (see Table 1) for the results shown in Fig. 6, the 1D approach still fails to reproduce the velocity field as determined by experiments and 2-D simulations. However, if BC's are extracted from the inner points of the domain (case denoted as " $L=0.4 D$ " in Fig. 6), the 
velocity profiles computed by 1-D and 2-D models match closely. Therefore, the domain of applicability of the 1-D approach in predicting the velocity field is smaller than what has been previously reported [3,17-20].

Figure 7 depicts the measured and computed axial velocity radial profiles at the burners' exits for both branches. Given that the symmetry of the profiles with respect to the centerline was better than $94 \%$ in all measurements, only half of the domain is shown; the lower branch velocities are plotted against negative radius values to aid the comparison with the upper branch profiles. The lower branch profile exhibits a larger dip at the lower burner exit because the stagnation point is closer to the lower burner; the observed dip results from pressure feedback from the stagnation point (e.g., [7,20,55]).

Using high-speed shadowgraph to capture the details of the ignition process, it was found that ignition at $X_{\mathrm{F}}=0.18(0.22)$ occurs at the upper (lower) branch. The observed 0.04 difference in $X_{\mathrm{F}}$ at which ignition occurs is rather significant in terms of the reactivity of the ignition kernel. Figure 8 depicts the spatial distributions of the hydrogen radical mole fractions $\left(X_{H}\right)$ along the centerline computed using 1-D approach which shows that there is a $31 \%$ difference between the maximum $X_{H}$ values for the two branches.

It is also a common practice to perform the flame ignition experiment in the counterflow configuration by keeping $X_{\mathrm{F}}$ constant and increasing $T_{\text {air }}$ until ignition is observed. To investigate if the observed difference in ignition propensity of the two branches is sensitive to the method of achieving ignition, $T_{\text {ign }}$ was measured for $X_{\mathrm{F}}=0.18$ and $X_{\mathrm{F}}=0.22$ with increasing $T_{\text {air }}$ and it was observed that ignition occurs at $T_{\text {air }}=926 \mathrm{~K}$ for both cases. Therefore, by changing $T_{\text {air }}$ the effects of bifurcation could not be captured. The low sensitivity of $T_{\text {ign }}$ to $X_{\mathrm{F}}$ is expected given that for the conditions considered in the present investigation, ignition is not controlled by fuel diffusion [33]. Figure 9 shows the variation of $T_{\mathrm{ign}}$ with $X_{\mathrm{F}}$ in which results of 1-D simulations are compared to the measurements. The 1-D results revealed that there is only $4 \mathrm{~K}$ difference between $T_{\text {ign }}$ 's at $X_{\mathrm{F}}=0.18$ and $X_{\mathrm{F}}=0.22$.

High-speed imaging of the flame luminosity revealed that ignition always initiates close to the centerline for both branches as shown in Fig. 10a. Subsequently, the resulting edge flame propagates radially through a path whose shape is similar to the stagnation plane before finally settling as a single-state stable non-premixed flame shown in Fig. 10c. These observations suggest that the lower branch is unstable in the presence of the flame, which is also evident from the transition of the lower branch flame (Fig. 10b) to the upper stable flame (Fig. 10c). A possible explanation is that in the presence of a flame, the lower branch flame becomes unstable due to buoyancy, which tends to push the stagnation plane wings upward at larger radial distances away from the centerline $[8,41]$. 
It should be noted that by comparing the features of Figs. $5 \mathrm{~b}$ and $10 \mathrm{c}$, the flame appears to be more planar compared to the shape of the light intensity discontinuity before ignition (Figs. 5b). This is due to the fact that the flame changes the characteristics of the flow field due to the heat release and buoyancy. Therefore judging the ideality of the flow field before ignition by the flame topology upon ignition can be misleading.

It was realized also that the reported bifurcation is sensitive to $T_{\text {air }}$ and $K$. At low $K$ and high $T_{\text {air }}$, only the upper branch was attainable, while the lower branch was realized only for high $K$ and low $T_{\text {air }}$ values, which could be caused by buoyancy [37]. Thus, given the wide range of $T_{\text {air }}$ and $K$ values that are encountered in counterflow flame ignition experiments, it is not clear what flow conditions will be prevailing at the pre-ignition states, and thus velocity measurements or shadowgraph visualization are required.

\subsection{Simulation results for geometry (a)}

2-D simulations were performed for geometry (a) for $X_{\mathrm{F}}=0.2$ and two stable states were found for the same BC's. Figure 11 depicts the temperature distribution along with the streamlines for the two stable branches. For comparison, the results of the lower branch solution are shown on the left while the results the results for upper branch are shown on the right, and they were separated by the stagnation streamline. At the junction of the coflow exits and disk walls, the flow separates creating recirculation zones. The upper separation bubble is smaller (bigger) than the lower one for the upper (lower) branch. The shape of the light intensity discontinuity captured by shadowgraph and shown in Fig. 5 is reproduced qualitatively by the temperature distribution in Fig. 11.

The spatial distributions of $X_{\mathrm{H}_{2}}$ and $X_{H}$ are shown in Fig. 12 for lower and upper branches. The maximum $X_{H}$ occurs at the centerline indicating the higher ignition propensity at the centerline in agreement with the results of Fig. 10a in which ignition was found experimentally to initiate at the centerline. All other minor species and products also maximize at the centerline implying that the ignition kernel is located at $r=0$. However, the $X_{\mathrm{H}_{2}}$ iso-contours curve at larger radii due to the parabolic nature of the axial velocity profile exiting the upper burner and the effect of elliptic pressure field on the lower burner exit. The most apparent difference between two branches is the shift of the ignition kernel due to the displacement of the stagnation point.

Computed $X_{H}$ profiles along the stagnation streamline are shown in Fig. 13 to further investigate the differences between the two solution branches. The maximum $X_{H}$ for the upper branch is $4 \%$ higher than the corresponding value for the lower branch, which could explain why lower-branch ignition happens at higher fuel concentrations experimentally. The $X_{H}$ profile computed using the 1-D opposed jet code are shown also in 
Fig. 13 and the maximum value exceeds by nearly 2.33 times those predicted by the 2-D simulations (for 1-D, Upper Branch case).

It should be noted also that central differencing schemes was used to discretize the 1-D domain as order of discretization has shown to be important when comparing 1-D and 2-D results [11]. Moreover, since comparisons of 1-D and 2-D results is only valid when rigorous quantification of discretization uncertainty for 2-D results is provided [11], the error bar for maximum $X_{H}$ value (as explained in detail in Appendix B of SPM) was quantified and shown in Fig. 13.

\subsection{Effects of jet exit parabolic temperature profile}

To further investigate the discrepancies between the results obtained by the 1-D and 2-D formulations, the computed temperature profiles along the centerline are shown in Fig. 14.

While the 1-D results predict a constant temperature from the exit of the top hot burner to the mixing layer, the 2-D simulations reveal a linear decrease of temperature, which is due to radial heat loss due to the parabolic temperature profile at the hot boundary (as shown in Fig. 15) which causes the heat to be transferred from the hotter gases in the center of the jet to the outer periphery as they are being convected axially.

Since it is known that such losses can affect significantly the interpretation of counterflow results [9], 1-D simulations were repeated accounting for the temperature drop; the temperature at the axial position at which the temperature drop deviates more than $1 \%$ from its linear trend $(z=0.43 D)$, was chosen as a new $T_{\text {air }}$ for the “adjusted T" simulations. The results are shown in Figs. 13 and 14. The discrepancy between the predicted maximum $X_{H}$ in the centerline was thus reduced from $232 \%$ to only $11 \%$, indicating that the most significant non-ideality of the experimental approach is the parabolicity of the temperature profile at the hot boundary whose effect on the reported results has not quantified in past studies [33,40,41]. Such parabolicity is required to ensure that ignition initiates at the centerline or else 1-D assumptions are violated [39-41]. On the other hand, the use of the 1-D formulation is frequently justified by asserting that although the measured velocity and temperature profiles have a parabolic shape in general, they exhibit uniformity in a region around the centerline as small as few millimeters (e.g., [33,44]).

As already shown by previous studies [4-10], in the counterflow configuration, axial velocity profiles must be of top-hat shape at the exit of the burners for the valid comparison of experimental data and results of 1-D codes. The present results suggest also that that the burner exit temperature must be uniform as well, and this is never the case in experiments. 
The observed sensitivity to the temperature profile is expected considering the exponential dependence of kinetics on temperature. Therefore, care should be taken when measuring temperatures at the hot boundary and it was determined that the thermocouple readings are sensitive to the thermocouple wire size such that not utilizing thermocouples with a very small bead will introduce intrusive measurement errors as big as $90 \mathrm{~K}$ as shown in Appendix C of SPM. It was found also that the intrusive effects can be minimized by using microthermocouples and measuring in the single jet configuration with fuel jet turned off as shown in Fig. 15.

The agreement between the uncorrected measured temperature and the 2-D numerical simulation results is less compared to velocities specifically at larger radii. This is mostly due to the extra requirement of the momentum balance at the burner exits for the numerical simulations, as discussed in Appendix B of SPM. Moreover, the fact that the nozzle walls were assumed to be adiabatic is not realistic. However, at the centerline, the temperatures after the correction do match and is equal to $T_{\text {air }}=926 \mathrm{~K}$.

Given the parabolic temperature profiles at the burner exit [33,40,41] and the monotonic increase of the curvature of the temperature profile at the centerline by increasing $T_{\text {air }}$ [40], it is essential to introduce corrections before comparing data to 1-D simulation results. Particularly, there is a need to estimate the temperature at the ignition kernel in the experiment in the presence of radial heat loss, $T_{\text {kernel}}$, to approximate the effective boundary temperature, $\left(T_{\text {air }}\right)_{\mathrm{eff}}$ for the 1-D simulations similar to what was performed in "adjusted T" simulations. $T_{\text {kernel }}$ can be approximated by solving the 1-D heat conduction problem in the radial direction. For given BC's, the ignition kernel position as marked by the maximum $X_{H}, z_{\text {kernel }}$, can be determined through 1-D opposed jet simulations. By integrating the axial velocity profile at the centerline obtained from 1-D simulations from $z=D$ to $z=z_{\text {kernel}}$, the time for the heat loss process, $t_{\text {loss }}$, can be computed. Subsequently, $T_{\text {kernel }}$ can be obtained by letting the known temperature profile at the hot boundary to evolve in time from its initial state to $t_{\text {loss. }}$. At last, since the boundary temperature is always slightly higher than $T_{\text {kernel, effective }}$ boundary temperature can be obtained such that $\left(T_{\text {air }}\right)_{\text {eff }}=T_{\text {kernel }} \times\left(\frac{T_{\text {air }}}{\left(T_{\text {kernel }}\right)_{\text {lossless }}}\right)$ where $\left(T_{\text {kernel }}\right)_{\text {lossless }}$ is the computed kernel temperature from 1-D opposed-jet simulation.

The success of this method was verified by applying on the upper branch solution. Evolution of the radial temperature profile from the 2-D numerical results at the exit of the air jet $(\mathrm{z}=D)$ was tracked utilizing the 1-D heat conduction simulations performed using laminarSMOKE code in a 1-D radial geometry and "corrected" results are compared to the 2-D ones as shown in Fig. 15. The temperature gradient was specified to be zero at $r=0$ while at $r=0.5 D$, temperature was chosen to be equal to the corresponding value from 2-D numerical results at the exit of the air jet. Results show only a difference of $2 \mathrm{~K}$ at the centerline between the "corrected" and the 2-D results at $z=z_{\text {kernel }}=0.39 D$. The discrepancy increases at larger radii due to radial convection of 
gases with higher temperature from the centerline, which causes the 2-D temperature profiles to reach higher values at larger radii. The 1-D opposed jet simulations were repeated using $\left(T_{\text {air }}\right)_{\text {eff }}$ obtained from the "corrected" solution; results are also plotted in Figs. 13. The "corrected" solution under-predicts the maximum $X_{H}$ only by $10 \%$, therefore the correction provides reasonable predictions considering the $\sim 7 \%$ discretization uncertainty of the 2-D numerical simulations.

Therefore, without having to perform expensive 2-D numerical simulations or extensive thermocouple measurements that is subject to intrusive errors, 1-D conduction simulations could provide corrected air boundary temperature to be used by 1-D opposed jet simulations whose results could be compared against the data. It should be mentioned also that since the 1-D simulations denoted as " $L=0.4 D$ " were performed using $T_{\text {air }}=917 \mathrm{~K}$, which is not a correct BC (as shown in Fig. 14), the predicted reactivity in the kernel is still off by $58 \%$ (as shown in Fig. 13) although the velocity profiles are captured by the simulations as demonstrated in Fig. 6. This is mostly due to the fact that the proper axial positions to apply the BC's for 1-D simulations is not known in priori because of the non-uniform temperature profile at the hot boundary.

\subsection{Simulation results for geometry $(b)$}

2-D numerical simulations were performed also for geometry (b) and it was realized that bifurcation also occurs despite having $\mathrm{BC}$ 's that conform to the assumptions of the 1-D formulation, which is mainly due to the interaction of the jets with the adjacent disk walls. It should be noted that since the 1-D model is derived for infinite-diameter burners, the introduction of finite diameters and adjacent walls could result in recirculation bubbles and eventually bifurcation. Figure 16 depicts the $X_{\mathrm{H}_{2}}$ distribution overlaid by the streamlines for the two stable branches. Similar to Fig. 11, the recirculation bubbles at the junction of coflow exits and disk walls have different sizes for the two branches indicating that the way the flow orients itself is different for the two branches as it convects outward. It is also apparent from Fig. 16 that there is considerable amount of $\mathrm{H}_{2}$ both in upper and lower recirculation zones for both solutions branches. This is mainly due to the large sizes of these recirculation bubbles, which cause considerable amount of mixing [37,56-58].

Figure 17 depicts the $X_{H}$ variation along the centerline for both branches and for the 1-D formulation. Results show that the bifurcation does not affect $X_{H}$ values and that the discrepancy between the 1-D and 2-D results is about $12 \%$. No noticeable difference was found between the axial velocity profiles at the centerline (shown in Appendix D of SPM) for the two branches and the 1-D formulation reproduces closely the flow 
velocities. This is expected as for geometry (b), axial velocities exhibit zero axial gradients at the burner exits complying with the "plug flow" assumptions of the 1-D formulation (e.g., [1,3,10,20]).

To further investigate the 2-D effects that caused the discrepancy in reactivity shown in Fig. 13 and 17, the assumption of the constant radial pressure curvature eigenvalue $J=(1 / \mathrm{r})(\partial \mathrm{P} / \partial \mathrm{r})$ invoked in the 1 -D formulation [1] was also tested. The typical results for obtained using 2-D simulations and those obtained using the 1-D formulation are shown in Fig. 18.

$J$ varies along the centerline for both geometries but more notably for geometry (a) for which 2-D effects are more profound. Nevertheless, the average over the whole axial domain for upper branch of geometry (a) is only $10 \%$ higher than the value predicted by the 1-D formulation the variations close to the stagnation point are smaller in accordance with the findings of previous studies $[5,16]$. Similar order of magnitude differences were observed for the upper branch of geometry (a). The discrepancy is much smaller for geometry (b) such that the maximum deviation of $J$ from the 1-D prediction is 5\%. Johnson et al. [11] showed that this maximum deviation for non-premixed $\mathrm{H}_{2}$ flames is $3 \%$ with $\mathrm{BC}$ 's similar to those used in geometry (b).

It was also found that the radial variations of the axial velocity and temperature profiles at an axial position close to the stagnation point had considerably different shapes for the two branches. As a result, the stagnation plane and kernel surface shapes are also different for the two branches. These results are discussed in Appendix D of SPM.

While the 1-D and 2-D results of the velocities and scalars along the centerline are in close agreement, the $X_{H}$ values predicted by 2-D simulations and shown in Fig. 19 indicate that the maximum reactivity is realized well outside the region of influence of the main jets for both branches. Additionally, the maximum $X_{H}$ value for the upper branch is $13 \%$ higher compared to the lower branch.

Such 2-D effects are due to the presence of the recirculation zones next to jet exits. Although the size of coflow annulus chosen for geometry (b) is larger than what is usually employed in experiments, it cannot isolate the main jets from interacting with the reactants fed back to the high temperature region by the recirculation bubbles as shown in Fig. 16. Specifically, since the bubble next to the upper burner exit is bigger for the lower branch, high temperature gases lose more heat as they recirculate through the bubble. Hence, the relatively hotter upper recirculation zone for the upper branch results in higher reactivity in that region as confirmed by the $X_{\mathrm{HO}_{2}}$ maps illustrated in Fig. 20, which reveals a profound difference in the distribution of $\mathrm{HO}_{2}($ an important pre-ignition radical) between the two branches. While the maximum $X_{\mathrm{HO}_{2}}$ occurs close to the wall at the upper recirculation zone for the upper branch, for the lower branch it is located between the two recirculation zones with the peak value being 10 times smaller than that of the upper branch. Hence, if 
geometry (b) is chosen in experiments, ignition will initiate at large radii. In this case, the comparison of the experimental results with prediction using the 1-D formulation may be meaningless.

The reason why such radical pool build up at large radii was not observed in the recirculation zones of geometry (a) is that the temperature drops rapidly at larger radii in that geometry due to the parabolic temperature profile of air jet in that geometry. The presence of the separation bubbles no matter how far they are pushed away from the main jets by the coflow, affects the way the flow field develops and modifies the species spatial distribution due to interactions of the main jest with the recirculation zones. In fact, the distribution of minor species could be modified in geometry (b) if ambient temperature coflow streams are utilized to eliminate the possibility of ignition initiation at larger radii, but on the other hand radial temperature gradients will be introduced and which are inconsistent with the assumptions of geometry (b).

\subsection{Simulation results for geometry $(c)$}

Only one stable state was found for geometry (c) as shown by temperature maps overlaid by entrainment streamlines in Fig. 21. Moreover, only one large recirculation zone forms between the top burner wall and upper outflow boundary. Due to larger size of this recirculation zone compared to the ones observed in geometry (b), the temperature inside the bubble is approximately the ambient temperature. High levels of buoyancy cause the main jet flow to rise upwards towards the upper open boundary and suppress the bifurcation tendency [37].

The variation of $X_{H}$ along the centerline of geometry (c) was also compared to the corresponding profiles for geometry (b) and 1-D results in Fig. 17; 1-D results match the ones from geometry (c) within the discretization uncertainty $(7 \%)$. Same order of agreement was observed for the axial velocities along the centerline as shown in Appendix D of SPM.

$X_{\mathrm{H}_{2}}$ isocontours overlaid by the $X_{H}$ maps for geometry (c) are shown in Fig. 22 revealing negligible 2-D effects. In contrast to geometry (b), $X_{H}$ maximizes at the centerline. The ignition kernel surface was determined to be nearly planar with a slight curvature towards the upper burner. All other intermediate radicals also peak in the region of jets interaction and iso-contours of the maxima are also flat along the radius. Due to the absence of recirculation zones, the main reactivity region is isolated between the jets. Moreover, the maximum variations of $J$ along the centerline is only $2 \%$ for geometry (c) as shown in Fig. 18, which is in agreement with findings of Johnson et al. [11] for unconfined non-premixed flames. Therefore, the results of 1D codes match closely those for geometry (c). 
Therefore the design of a counterflow flame ignition experiment should be such that it matches the conditions of geometry (c) as close as possible which is the burner design proposed by Niemann et al. [10] with no solid surfaces close to jets exists. Solid surfaces close to the jet exits must be removed or trimmed wherever possible to avoid recirculation [22], which can result in bi-stable states. It should also be noted that the presence of vortices could introduce 3-D complications at higher Re. The axisymmetric vortex rings can lose stability by side jet generation phenomena [59] and the flow can lose its axisymmetry by azimuthal disturbances [60]. This is particularly important since the requirement of eliminating vortices is not always fulfilled in the burner designs (e.g., [8,38]) and it has been reported to cause 2-D complications in one case [38].

It is expected that for a given $T_{\text {air }}$ and $K$ and for momentum-balanced jets, there is a maximum allowable thickness of disk walls i.e., cooling jacket thickness (denoted as $\delta$ in Fig. 2.) above which recirculation zones form close to the jet exists. These recirculation bubbles are susceptible to undesirable instabilities. Therefore, it is worthwhile to perform a parametric study to investigate the effect of $\delta$ on the bifurcation propensity. Moreover, since the existence of bi-stable states depend on both $T_{\text {air }}$ and $K$ it would be useful if bifurcation maps could be generated to quantify the effects of each variable.

\section{Concluding remarks}

The one-dimensional assumptions invoked in counterflow flame ignition experiments were assessed experimentally and computationally for non-premixed $\mathrm{H}_{2}$ flames. The novelty of the present study is that the combined effects of momentum and thermal boundary layers on the flow field established in counterflow configurations have been considered.

For the first time, the existence of two stable states of the pre-ignition flow field was identified experimentally for a given set of boundary conditions. Ignition was observed to occur at different fuel mole fractions for the two stable states. Two-dimensional (axisymmetric) numerical simulations reproduced the two stable solutions that are characterized by different distributions of velocities and scalars and thus different ignition propensities. The solid surfaces next to the jets exits were identified to cause the bifurcation due to flow separation.

It was determined also that one-dimensional simulations overestimate notably the reactivity around the centerline compared to the results of the two-dimensional simulations. This difference is mainly caused by the non-uniform radial temperature profiles that are unavoidable in ignition experiments. A novel correction method based on simple numerical heat conduction calculations was developed to reduce the discrepancies. It 
was shown that by utilizing an effective hot air boundary temperature as the boundary condition for the onedimensional approach, the two-dimensional results could be recovered.

To isolate two-dimensional effects, axisymmetric simulations were carried out also for ideal boundary conditions that conform to those of the one-dimensional formulation with the only exception being the use of finite burner diameter. While the two formulations result in similar velocities and scalar distributions along the centerline as expected, it was found that radicals build up at larger radii due to the jet-wall interaction. Therefore, ignition can initiate off-center. This undesirable effect can be eliminated by removing solid surfaces from the jets vicinity.

It is recommended that the flow behavior observed only at pre-ignition states needs to be considered in the design of the experimental apparatus, and that direct high-speed visualization of the entire flow-field is required in order to evaluate potential errors when using standard one-dimensional codes to model and interpret the data.

\section{Acknowledgements}

This material is based upon work supported as part of the CEFRC, an Energy Frontier Research Center funded by the U.S. Department of Energy, Office of Science, and Office of Basic Energy Sciences under Award Number DE-SC0001198. The numerical simulations were carried out using the Extreme Science and Engineering Discovery Environment (XSEDE), which is supported by National Science Foundation grant number TG-CTS140012. The authors would like to gratefully acknowledge the assistance of Dr. Vyaas Gururajan for his feedback and technical support with the numerical simulations, and of Dr. Francesco Carbone for the velocity measurements.

\section{References}

[1] R.J. Kee, J.A. Miller, G.H. Evans, G. Dixon-Lewis, A computational model of the structure and extinction of trained, opposed flow, premixed methane-air flames, Symp. (Int.) Combust. 22 (1988) 14791494.

[2] A. Ern, M.D. Smooke, Vorticity-velocity formulation for three-dimensional steady compressible flow, J. Comput. Phys. 105 (1993) 58-71.

[3] H.K. Chelliah, C.K. Law, T. Ueda, M.D. Smooke, F.A. Williams, An experimental and theoretical investigation of the dilution, pressure and flow-field effects on the extinction condition of methane-airnitrogen diffusion flames, Symp. (Int.) Combust. 23 (1990) 503-511. 
[4] Y.M. Kim, H-J. Kim, Multidimensional effects on structure and extinction process of counterflow nonpremixed hydrogen-air flames, Combust. Sci. Tech. 137(1998) 51-80.

[5] C.E. Frouzakis, J. Lee, A.G. Tomboulides, K. Boulouchos, Two-dimensional direct numerical simulation of opposed-jet hydrogen-air diffusion flame, Symp. (Int.) Combust. 27 (1998) 571-577.

[6] W.-C. Park, A. Hamins, Investigation of velocity boundary conditions in counterflow flames, J. Mech. Sci. Technol. 16 (2) (2002) 262-269.

[7] E. Korusoy, J.H. Whitelaw, Inviscid, laminar and turbulent opposed flows, Int. J. Numer. Meth. Fl. 46 (2004) 1069-1098.

[8] G. Amantini, J.H. Frank, M.D. Smooke, A. Gomez, Computational and experimental study of steady axisymmetric non-premixed methane counterflow flames, Combust. Theory and Model. 11 (2007) 47-72.

[9] V. Mittal, H. Pitsch, F.N. Egolfopoulos, Assessment of counterflow to measure laminar burning velocities using direct numerical simulation, Combust. Theory and Model. 16 (2012) 419-433.

[10] U. Niemann, K. Seshadri, F.A. Williams, Accuracies of laminar counterflow flame experiments, Combust. Flame 162 (2015) 1540-1549.

[11] R.F. Johnson, A.C. VanDine, G.L. Esposito, H.K. Chelliah, On the Axisymmetric Counterflow Flame Simulations: Is There an Optimal Nozzle Diameter and Separation Distance to Apply Quasi OneDimensional Theory?, Combust. Sci. Tech. 187 (2015) 37-59.

[12] J.M. Bergthorson, D.G. Goodwin, P.E. Dimotakis, Particle streak velocimetry and CH laser-induced fluorescence diagnostics in strained, premixed, methane-air flames, Proc. Combust. Inst. 30 (2005) 16371644.

[13] P. Versailles, J.M. Bergthorson, Optimized Laminar Axisymmetrical Nozzle Design Using a Numerically Validated Thwaites Method, J. Fluids Eng. 134 (2012).

[14] P. Versailles, G.M. Watson, A.C. Lipardi, J.M. Bergthorson, Quantitative CH measurements in atmospheric-pressure, premixed flames of $\mathrm{C}_{1}-\mathrm{C}_{4}$ alkanes, Combust. Flame 165 (2016) 109-124.

[15] N. Bouvet, D. Davidenko, C. Chauveau, L. Pillier, Y. Yoon, On the simulation of laminar strained flames in stagnation flows: 1D and 2D approaches versus experiments, Combust. Flame 161 (2014) 438-452.

[16] J.M. Bergthorson, K. Sone, T.W. Mattner, P.E. Dimotakis, D.G. Goodwin, D.I. Meiron, Impinging laminar jets at moderate Reynolds numbers and separation distances. Phys. Rev. E 72 (2005) 066307.

[17] J.M. Bergthorson, S.D. Salusbury, P.E. Dimotakis, Experiments and modelling of premixed laminar stagnation flame hydrodynamics, J. Fluid Mech. 681 (2011) 340-369. 
[18] L.W. Kostiuk, K.N. Bray, R.K. Cheng, Experimental study of premixed turbulent combustion in opposed streams. Part I-Nonreacting flow field, Combust. Flame 92 (1993) 377-395.

[19] G. Pellett, K. Isaac, W. Humphreys, L. Garttrell, W. Roberts, C. Dancey, G. Northam, Velocity and thermal structure, and strain-induced extinction of 14 to $100 \%$ hydrogen-air counterflow diffusion flames, Combust. Flame 112 (1998) 575-592.

[20] B.G. Sarnacki, G. Esposito, R.H. Krauss, H.K. Chelliah, Extinction limits and associated uncertainties of nonpremixed counterflow flames of methane, ethylene, propylene and n-butane in air, Combust. Flame 159 (2012) 1026-1043.

[21] J.L. Fox, G.W. Morgan, On the stability of some flows of an ideal fluid with free surfaces, Brown Univ. Providence RI DIV of Applied Mathematics (1952), paper TR-2.

[22] J.C. Rolon, D. Veynante, J.P. Martin, Counter jet stagnation flows, Exp. Fluids 11 (1991) 313-324.

[23] R.P. Pawlowski, A.G. Salinger, J.N. Shadid, T.J. Mountziaris, Bifurcation and stability analysis of laminar isothermal counterflowing jets, J. Fluid Mech. 551 (2006) 117-139.

[24] R.P. Pawlowski, Numerical studies of complex reacting Flows, Ph.D. thesis, 2000.

[25] A. Ciani, W. Kreutner, C.E. Frouzakis, K. Lust, G. Coppola, K. Boulouchos, An experimental and numerical study of the structure and stability of laminar opposed-jet flows, Comput. Fluids 39 (2010) 114124.

[26] S.T. Strogatz, Nonlinear Dynamics and Chaos: with applications to physics, biology, chemistry, and engineering, Westview Press, 2000, pp. 44-93.

[27] R.M. Feran, T. Mullin, K.A. Cliffe, Nonlinear flow phenomena in a symmetric sudden expansion, J. Fluid Mech. 221 (1990) 596-608.

[28] D.A. Hammond, L.G. Redekopp, Local and global instability properties of separation bubbles, Eur. J. Mech.,B/Fluids 17 (1998) 145-164.

[29] P.G. Drazin, W.H. Reid, Hydrodynamic stability, Cambridge University Press, 2004, pp. 126-144.

[30] O. Park, P.S. Veloo, H. Burbano, F.N. Egolfopoulos, Studies of premixed and non-premixed hydrogen flames, Combust. Flame 162 (2015) 1078-1094.

[31] N. Liu, C. Ji, F.N. Egolfopoulos, Ignition of non-premixed $\mathrm{C}_{3}-\mathrm{C}_{12}$ n-alkane flames, Combust. Flame 159 (2012) 465-475.

[32] R. Seiser, K. Seshadri, E. Piskernik, A. Linán, Ignition in the viscous layer between counterflowing streams: Asymptotic theory with comparison to experiments, Combust. Flame 122 (2000) 339-349.

[33] C.G. Fotache, Ignition in convestive-diffusive systems, Ph.D. thesis, 1997. 
[34] C.G. Fotache, T.G. Kreutz, D.L. Zhu, C.K. Law, An Experimental Study of Ignition in Nonpremixed Counterflowing Hydrogen versus Heated Air, Combust. Sci. Technol. 109 (1995) 373-393.

[35] V. Gupta, S.A. Safvi, T.J. Mountziaris, Gas-phase decomposition kinetics in a wall-less environment using a counterflow jet reactor: design and feasibility studies, Ind. Eng. Chem. Res. 35 (1996) 3248-3255.

[36] W. Zhang, Z. Chai, Z. Guo, B. Shi, Lattice Boltzmann Study of Flow and Temperature Structures of NonIsothermal Laminar Impinging Streams, Commun. Comput. Phys. 13 (2013) 835-850.

[37] N. Hassan, S.A. Khan, Two-dimensional interactions of non-isothermal counter-flowing streams in an adiabatic channel with aiding and opposing buoyancy, Int. J. Heat Mass Transfer 54 (2011) 1150-1167.

[38] A. Ciani, W. Kreutner, W. Hubschmid, C.E. Frouzakis, K. Boulouchos, Experimental investigation of the morphology and stability of diffusion and edge flames in an opposed jet burner, Combust. Flame 150 (2007) 188-200.

[39] S. Humer, K. Seshadri, R. Seiser, Combustion of jet fuels and its surrogates in laminar nonuniform flows, $5^{\text {th }}$ US Combustion Meeting (2007), paper E09.

[40] A. Ansari, F.N. Egolfopoulos, Further considerations of flame ignition in counterflow configuration, Western States Section of the Combustion Institute Spring Meeting (2014), paper 087LF-0051.

[41] K.B. Brady, X. Hui, C.J. Sung, K.E. Niemeyer, Counterflow ignition of $n$-butanol at atmospheric and elevated pressures, Combust. Flame 162 (2015) 3596-3611.

[42] O. Park, P.S. Veloo, N. Liu, F.N. Egolfopoulos, Combustion characteristics of alternative gaseous fuels. Proc. Combust. Inst. 33 (2011) 887-894.

[43] Y. Dong, C.M. Vagelopoulos, G.R. Spedding, F.N. Egolfopoulos, Measurement of laminar flame speeds through digital particle image velocimetry: mixtures of methane and ethane with hydrogen, oxygen, nitrogen, and helium, Proc. Combust. Inst. 29 (2002) 1419-1426.

[44] C.S. Yoo, J.H. Chen, J.H. Frank, A numerical study of transient ignition and flame characteristics of diluted hydrogen versus heated air in counterflow, Combust. Flame 156 (2009) 140-151.

[45] X.L. Zheng, J.D. Blouch, D.L. Zhu, T.G. Kreutz, C.K. Law, Ignition of premixed hydrogen/air by heated counterflow, Proc. Combust. Inst. 29 (2002) 1637-1643.

[46] A. Cuoci, A. Frassoldati, T. Faravelli, E. Ranzi, A computational tool for the detailed kinetic modeling of laminar flames: Application to $\mathrm{C}_{2} \mathrm{H}_{4} / \mathrm{CH}_{4}$ coflow flames, Combust. Flame 160 (2013) 870-886.

[47] http://www.openfoam.org.

[48] H.G. Weller, G. Tabor, H. Jasak, and C. Fureby, A tensorial approach to computational continuum mechanics using object-oriented techniques, Computers in Physics 12 (1998) 620-631. 
[49] A. Cuoci, A. Frassoldati, T. Faravelli, E. Ranzi, Numerical modeling of laminar flames with detailed kinetics based on the operator-splitting method, Energ. Fuel. 27 (2013) 7730-7753.

[50] V. Gururajan, F.N. Egolfopoulos, Direct numerical simulations of probe effects in low-pressure flame sampling, Proc. Combust. Inst. 35 (2015) 821-829.

[51] H.W. Wang, X. You, A.V. Joshi, S.G. Davis, A. Laskin, F.N. Egolfopoulos, C.K. Law, USC-Mech Version II. High-temperature combustion reaction model of $\mathrm{H}_{2} / \mathrm{CO} / \mathrm{C} 1-\mathrm{C} 4$ Compounds. http://ignis.usc.edu/USC-Mech-II.htm

[52] A.E. Lutz, R.J. Kee, J.F. Grcar, F.M. Rupley, OPPDIF: A FORTRAN program for computing opposedflow diffusion flames, Snadia Report, SAND96-8243, Sandia National Laboratories, 1997.

[53] M. Nishioka, C.K. Law, T. Takeno, A flame-controlling continuation method for generating S-curve responses with detailed chemistry, Combust. Flame 104 (1996) 328-342.

[54] F.N. Egolfopoulos, P.E. Dimotakis, Non-premixed hydrocarbon ignition at high strain rates, Symp. (Int.) Combust. 27 (1998) 641-648.

[55] C.M. Vagelopoulos, F.N. Egolfopoulos, Direct experimental determination of laminar flame speeds, Symp. (Int.) Combust. 27 (1998) 513-519.

[56] S.M. Hosseinalipour, A.S. Mujumdar, Flow and thermal characteristics of steady two dimensional confined laminar opposing jets: Part I. Equal jets, Int. Commun. Heat Mass 24 (1997) 27-38.

[57] S. Devahastin, A.S. Mujumdar, A numerical study of flow and mixing characteristics of laminar confined impinging streams, Chem. Eng. J. 85 (2002) 215-223.

[58] S.J. Wang, S. Devahastin, A.S. Mujumdar, Effect of temperature difference on flow and mixing characteristics of laminar confined opposing jets, Appl. Therm. Eng. 26 (2006) 519-529.

[59] P.A. Monkewitz, D.W. Bechert, B. Barsikow, B. Lehmann, Self-excited oscillations and mixing in a heated round jet. J. Fluid Mech. 213 (1990) 611-639.

[60] G. Coppola, A. Gomez, Experimental study of highly turbulent isothermal opposed-jet flows, Phys. Fluids 22 (2010) 105101. 
Table 1. Summary of cases investigated computationally.

\begin{tabular}{|c|c|c|c|}
\hline \multirow[b]{2}{*}{ Geometry } & \multirow{2}{*}{$\begin{array}{l}\underline{\text { 2-D simulation }} \\
\underline{\text { name }}\end{array}$} & \multicolumn{2}{|r|}{ 1-D simulation } \\
\hline & & $\underline{\text { Name }}$ & $\begin{array}{c}\text { Boundary conditions for scalars, axial velocity and } \\
\text { its gradient }\end{array}$ \\
\hline \multirow{5}{*}{ (a) } & \multirow{4}{*}{ 2-D, Upper Branch } & 1-D, Upper Branch & Extracted from 2-D, Upper Branch at $z=0 \& z=D$ \\
\hline & & $\begin{array}{l}1-\mathrm{D}(L=0.4 D), \text { Upper } \\
\text { Branch }\end{array}$ & $\begin{array}{c}\text { Extracted from 2-D, Upper Branch at } z=0.15 D \& z=0.55 D \\
\text { (with domain size of } L=0.4 D \text { ) }\end{array}$ \\
\hline & & $\begin{array}{l}\text { 1-D (adjusted T), Upper } \\
\text { Branch }\end{array}$ & $\begin{array}{c}\text { Extracted from 2-D, Upper Branch at } z=0 \& z=D \text { except } T_{\text {air }}=917 \mathrm{~K} \\
\text { (value at } z=0.43 D \text { ) }\end{array}$ \\
\hline & & $\begin{array}{l}\text { 1-D (corrected), Upper } \\
\text { Branch }\end{array}$ & $\begin{array}{l}\text { Extracted from 2-D, Upper Branch at } z=0 \& z=D \text { except } T_{\text {air }}= \\
\qquad\left(T_{\text {air }}\right)_{\text {eff }} \text { (obtained from correction procedure) }\end{array}$ \\
\hline & 2-D, Lower Branch & 1-D, Lower Branch & Extracted from 2-D, Lower Branch at $z=0 \& z=D$ \\
\hline \multirow{2}{*}{ (b) } & $\begin{array}{l}\text { 2-D, geometry (b), } \\
\text { Upper Branch }\end{array}$ & \multirow{3}{*}{$1-\mathrm{D}$} & \multirow{3}{*}{ Specified axial velocity and scalars, zero axial velocity gradient } \\
\hline & $\begin{array}{c}\text { 2-D, geometry (b), } \\
\text { Lower Branch }\end{array}$ & & \\
\hline (c) & 2-D, geometry (c) & & \\
\hline
\end{tabular}

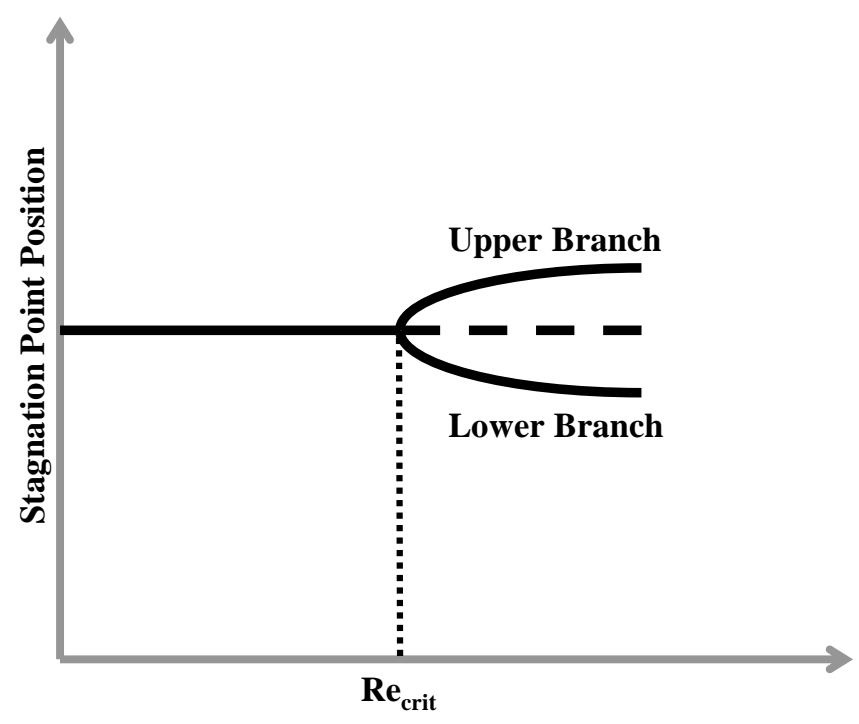

Fig. 1. Schematic of a typical stability diagram for counterflowing jets $[23,25]$. The two stable solutions are distinct after the bifurcation. 


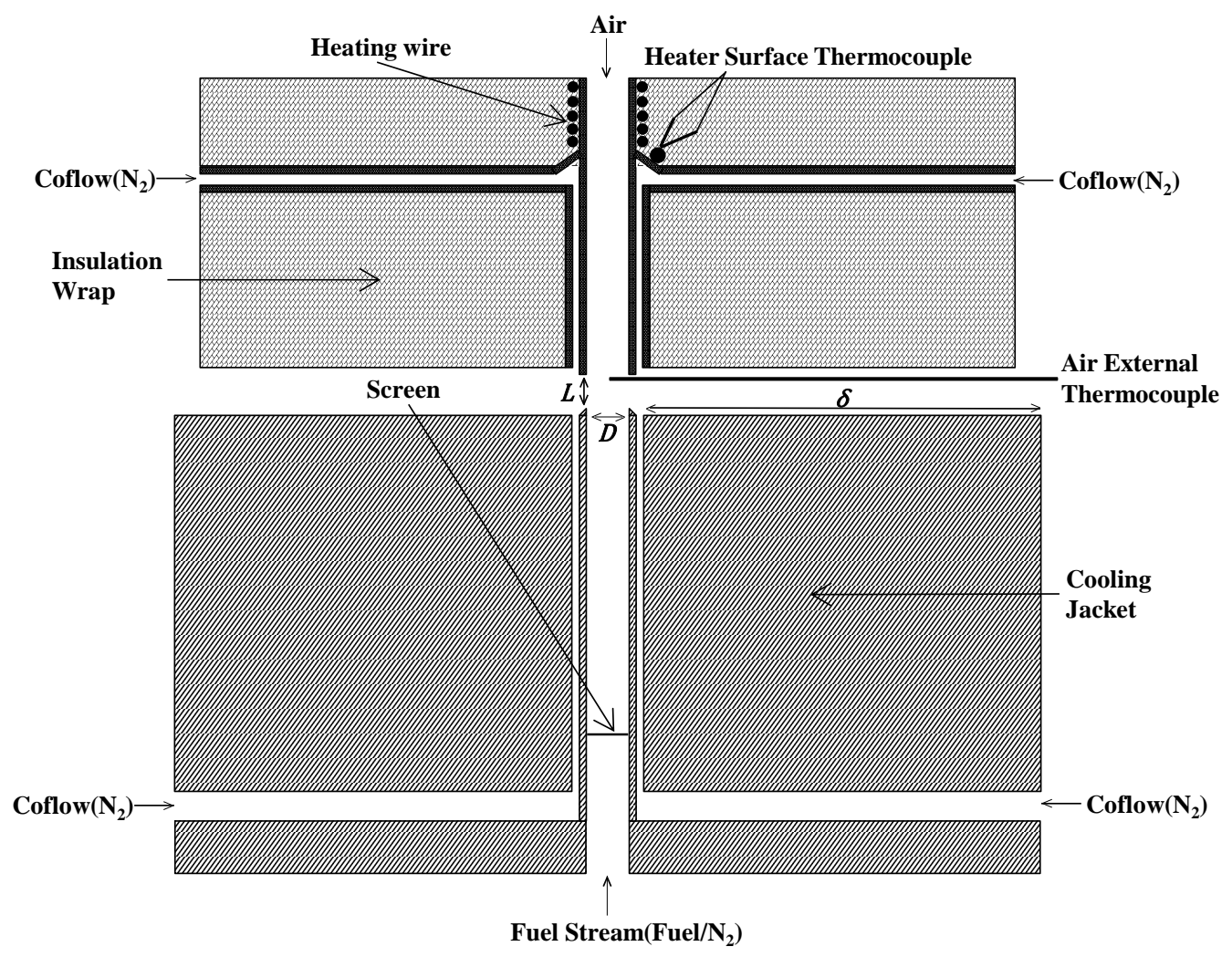

Fig. 2. Schematic of the counterflow ignition burner assembly.

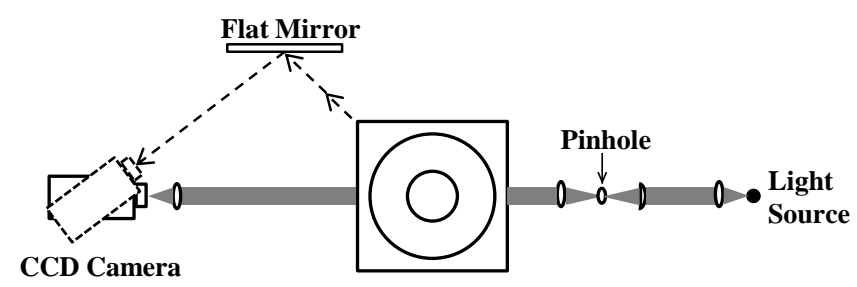

Fig. 3. Top view schematic of the visualization configuration. 


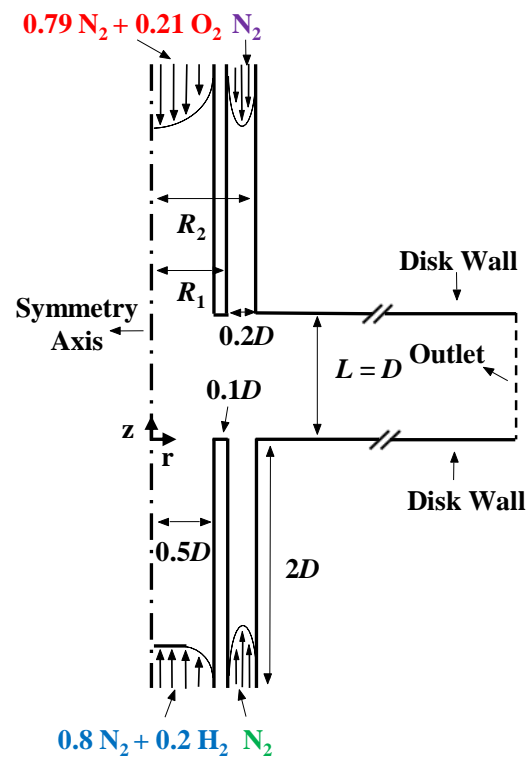

(a)

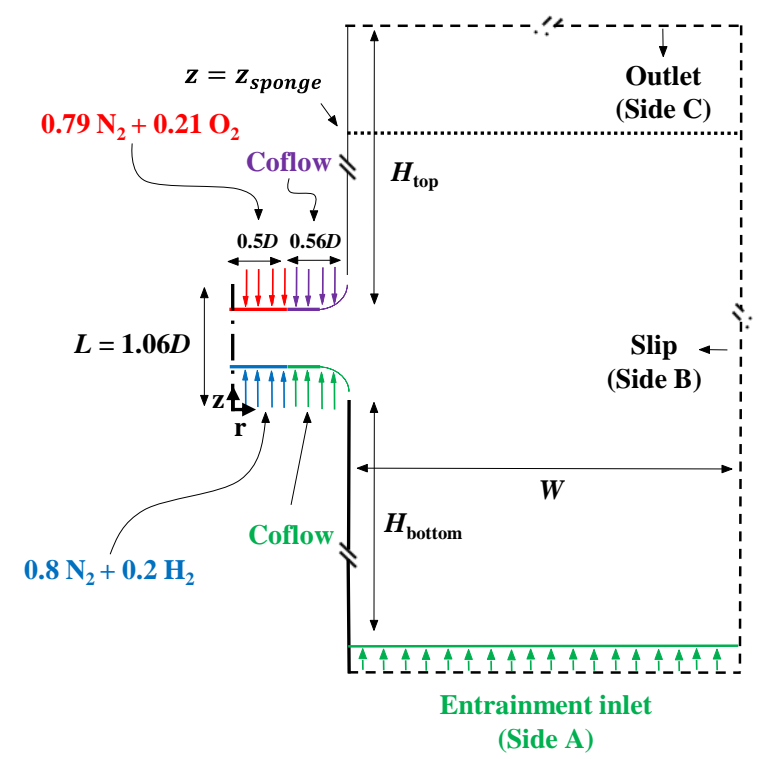

(b)

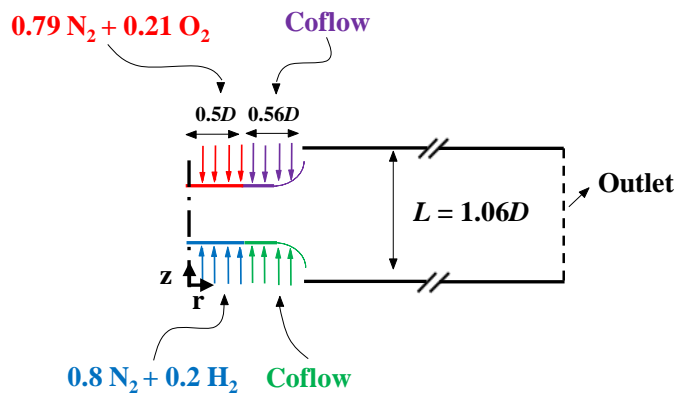

(c)

Fig. 4. Schematics of the geometries used in 2-D simulations; Solid boundary lines represent walls; a) geometry (a); b) geometry (c); c) geometry (b).

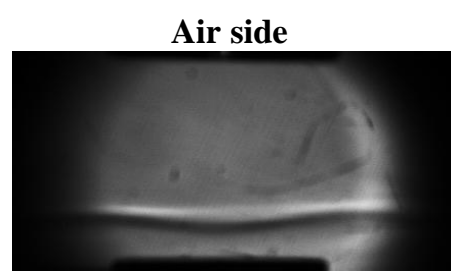

Fuel side

(a)

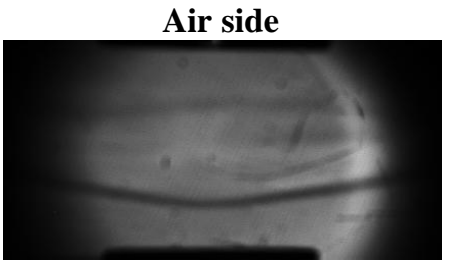

Fuel side

(b)

Fig. 5. Shadowgraph images of pre-ignition states showing two possible steady states for the same boundary conditions; (a) Lower branch; (b) Upper branch. 


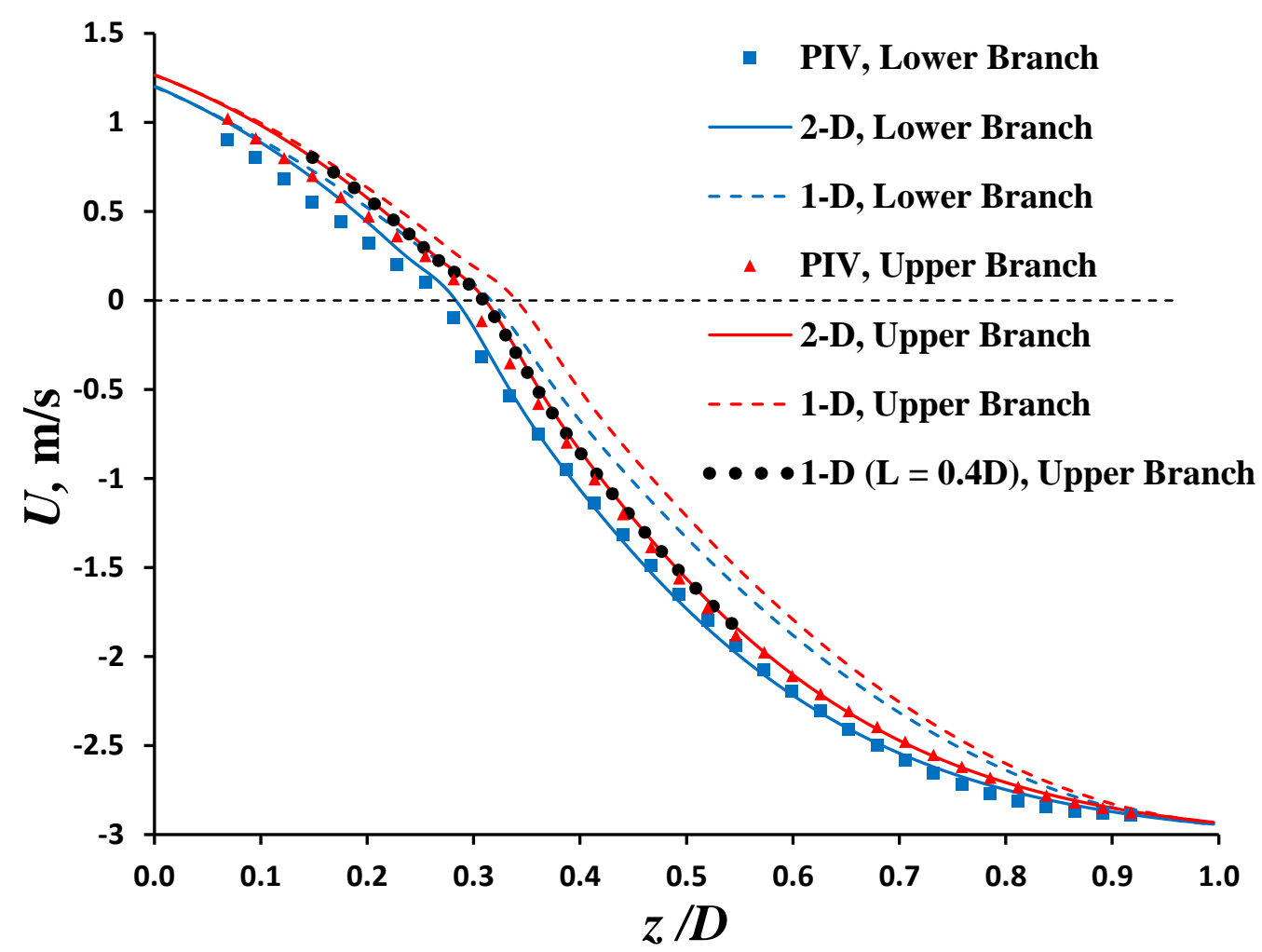

Fig. 6. Comparison between measured and computed axial velocity profiles at the stagnation streamline for geometry (a).

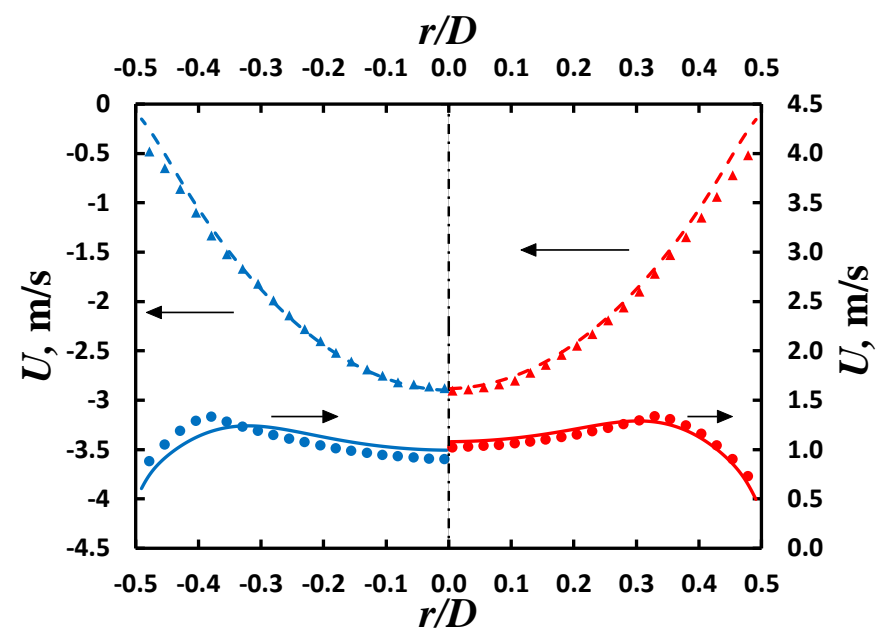

Fig. 7. Comparison between measured and computed radial profiles of axial velocity at the burners exits for geometry (a); ( $\mathbf{\Delta})$ and $(---) z=0.93 D,(\bullet)$ and $(-) z=0.07 D$. Symbols: experimental data; Lines: 2-D simulation results; Blue: Lower branch; Red: Upper branch. 


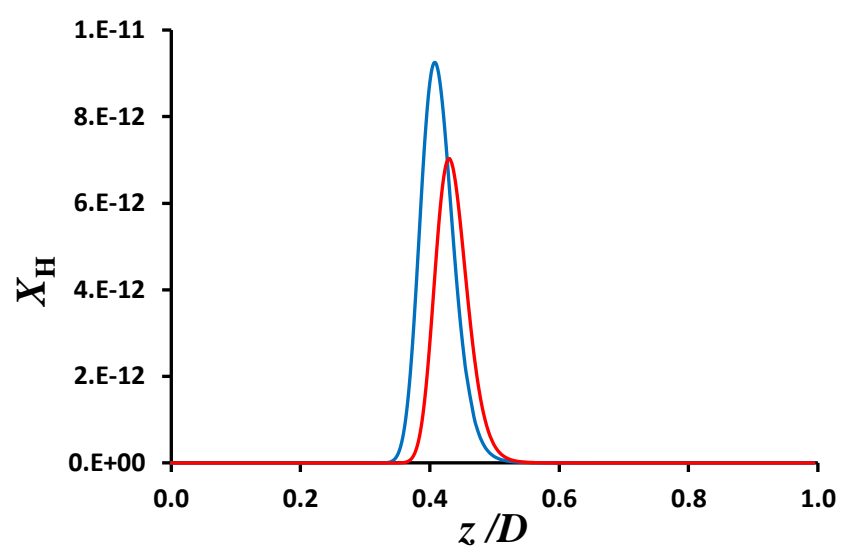

Fig. 8. Computed $X_{H}$ along the stagnation streamline of geometry (a) using 1-D approach; Red: Upper Branch, $X_{\mathrm{F}}=0.18$; Blue: Lower Branch, $X_{\mathrm{F}}=0.22$.

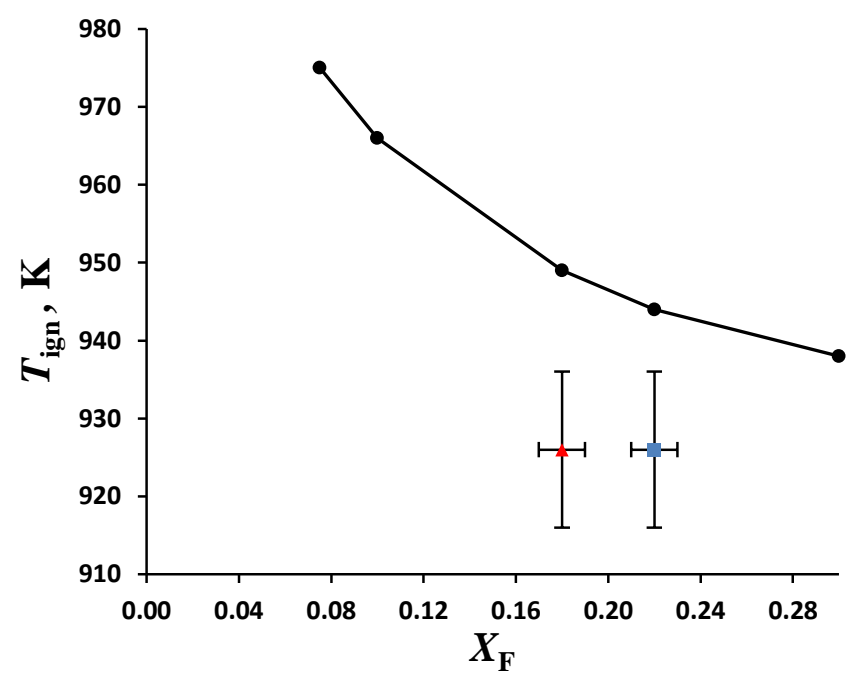

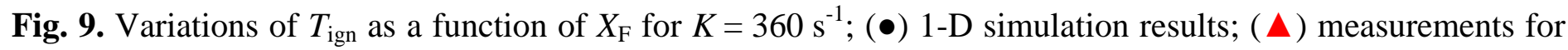
the upper branch; ( $\square$ ) measurements for the lower branch.

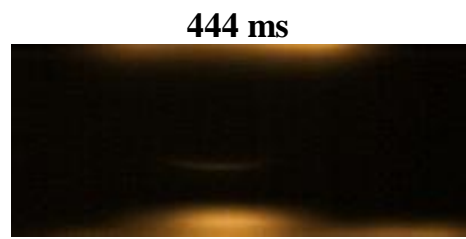

(a)

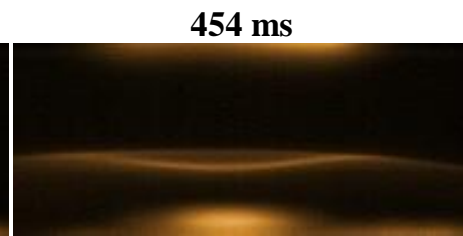

(b)
$728 \mathrm{~ms}$

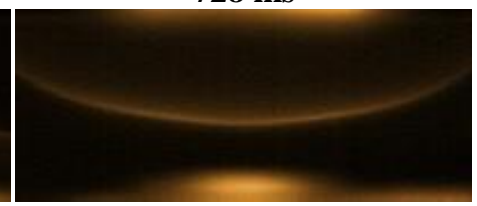

(c)

Fig. 10. High-speed images of ignition for $\mathrm{H}_{2}$ non-premixed flame at different times after increasing $X_{\mathrm{F}}$ to the ignition value for pre-ignition conditions corresponding to the lower branch; (a) ignition kernel formation; (b) unstable lower branch flame; (c) stable upper branch flame. 


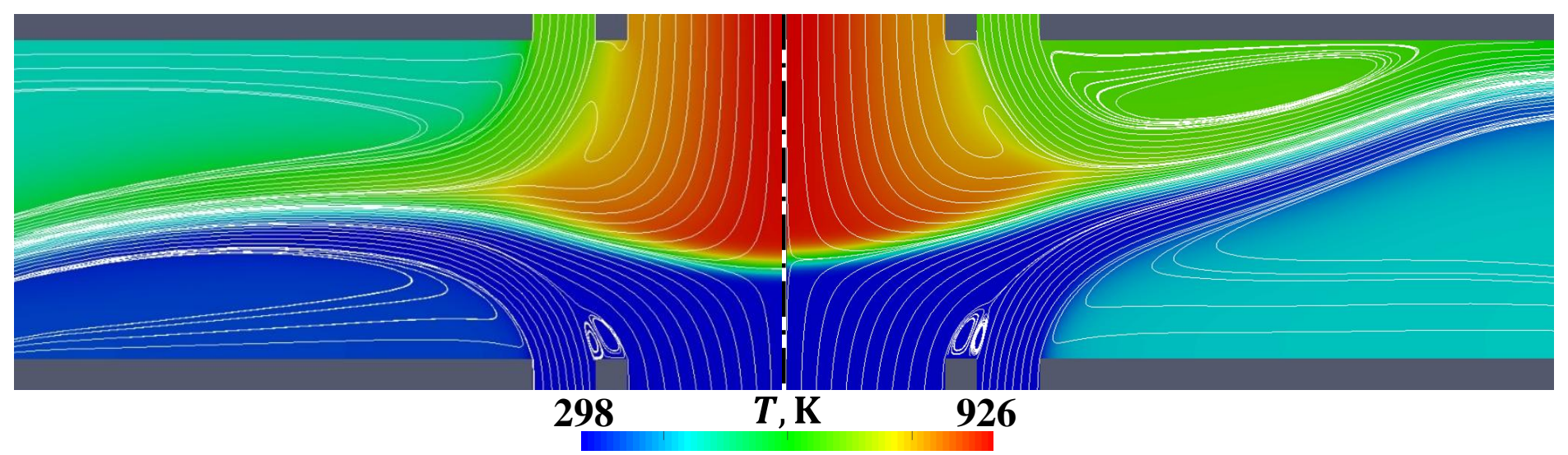

Fig. 11. Comparison between computed temperature maps superimposed by the streamlines for two stable solutions of geometry (a); Left: lower branch; Right: upper branch.

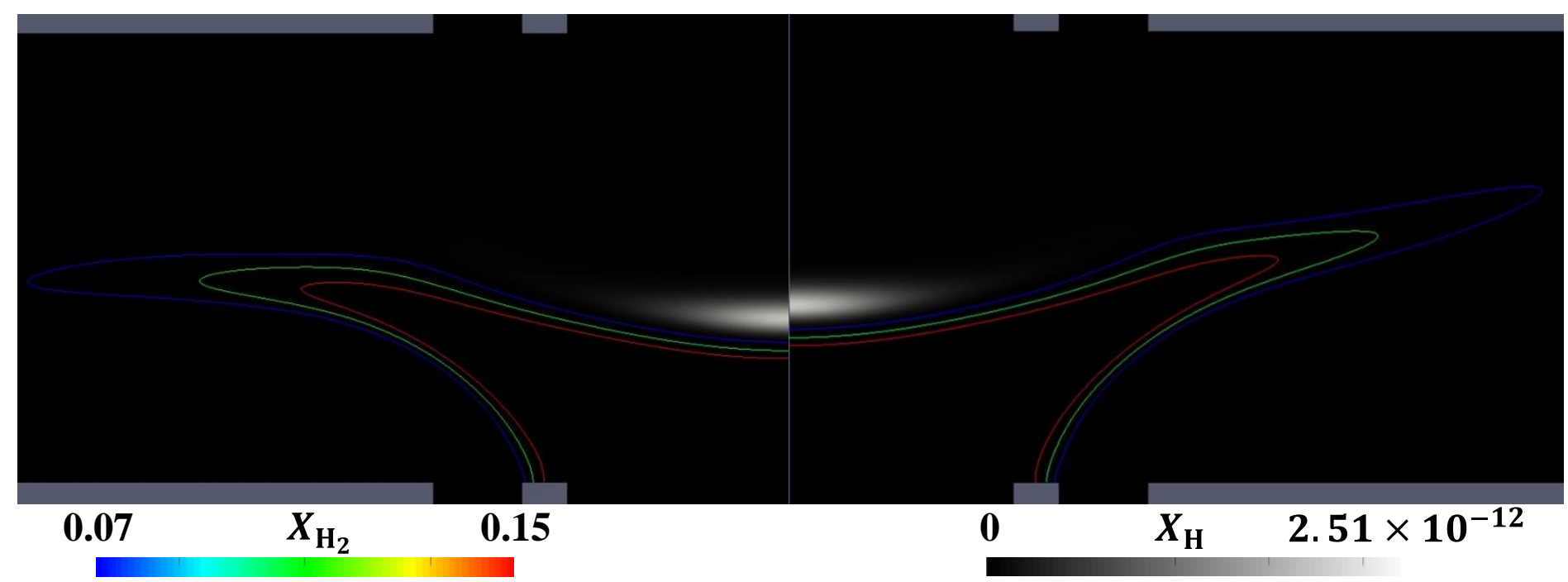

Fig. 12. Computed $\boldsymbol{X}_{\boldsymbol{H}}$ maps overlaid by $\boldsymbol{X}_{\mathbf{H}_{2}}$ iso-contours for the two stable solutions of geometry (a); Left: lower branch; Right: upper branch. 


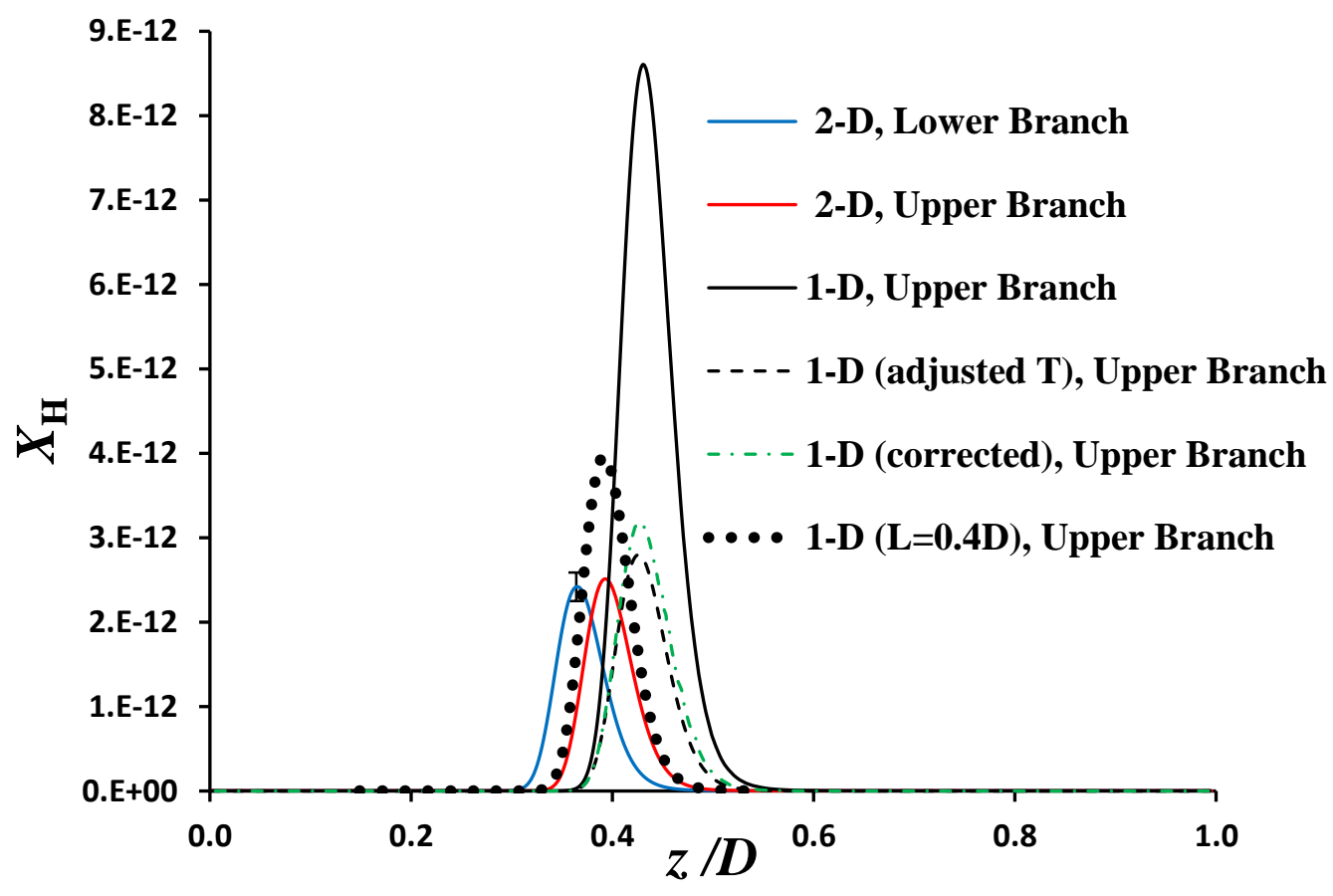

Fig. 13. Computed $\boldsymbol{X}_{\boldsymbol{H}}$ along the stagnation streamline of geometry (a).

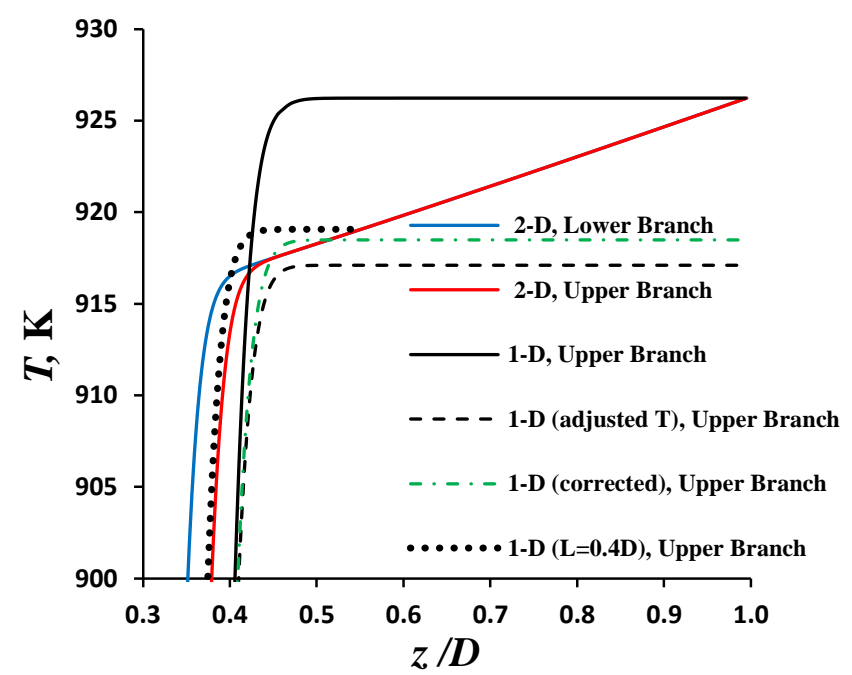

Fig. 14. Computed temperature profiles along the stagnation streamline of geometry (a). 


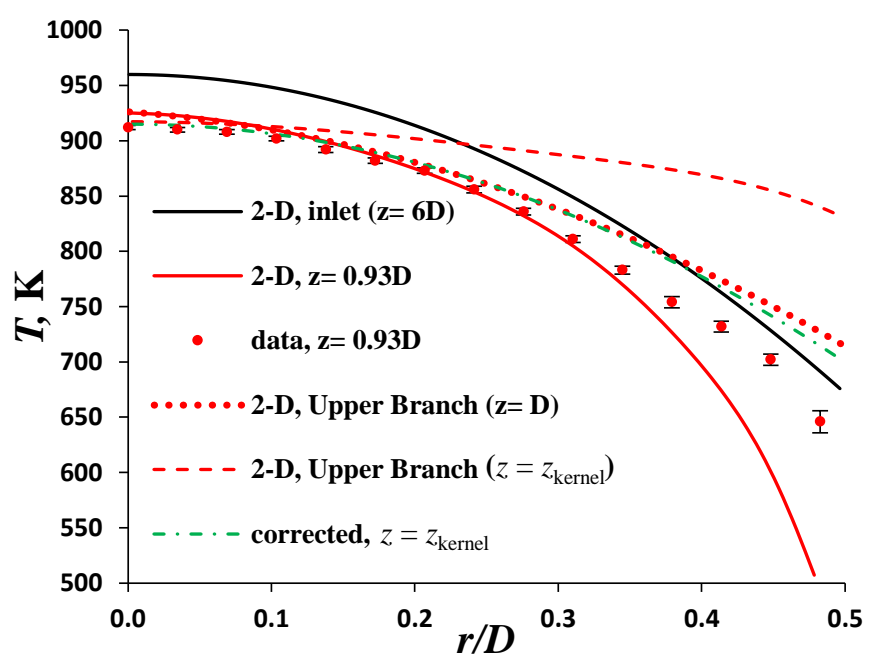

Fig. 15. Comparison between uncorrected data and computed radial profiles of temperature of geometry (a); profiles at $z=0.93 D$ are obtained in the absence of the fuel-jet.

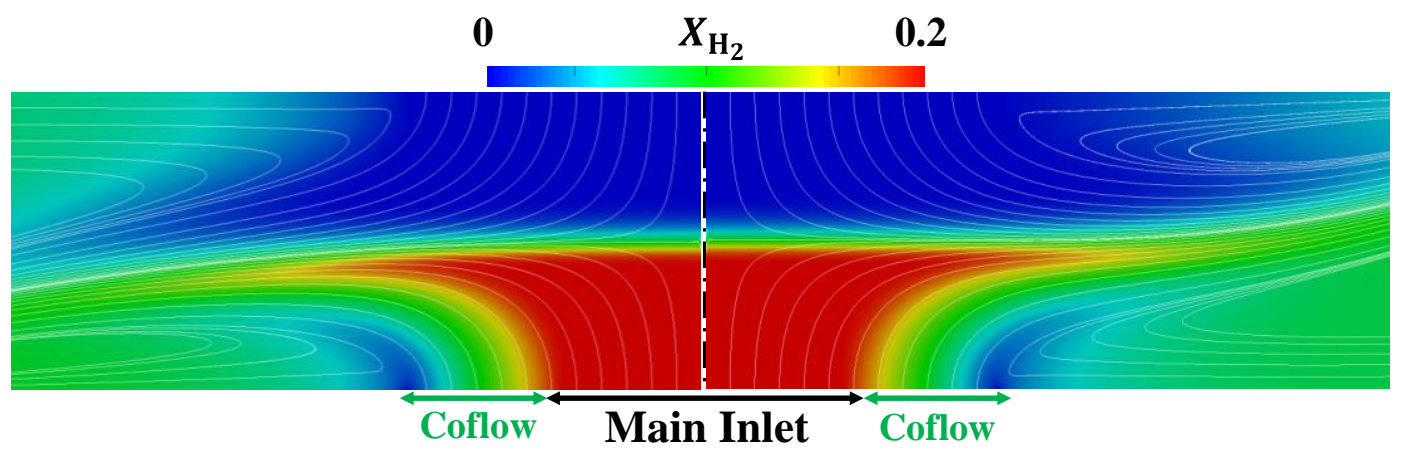

Fig. 16. Comparison between computed $\boldsymbol{X}_{\mathbf{H}_{2}}$ maps overlaid by the streamlines for two stable solutions of geometry (b); Left: lower branch; Right: upper branch. 


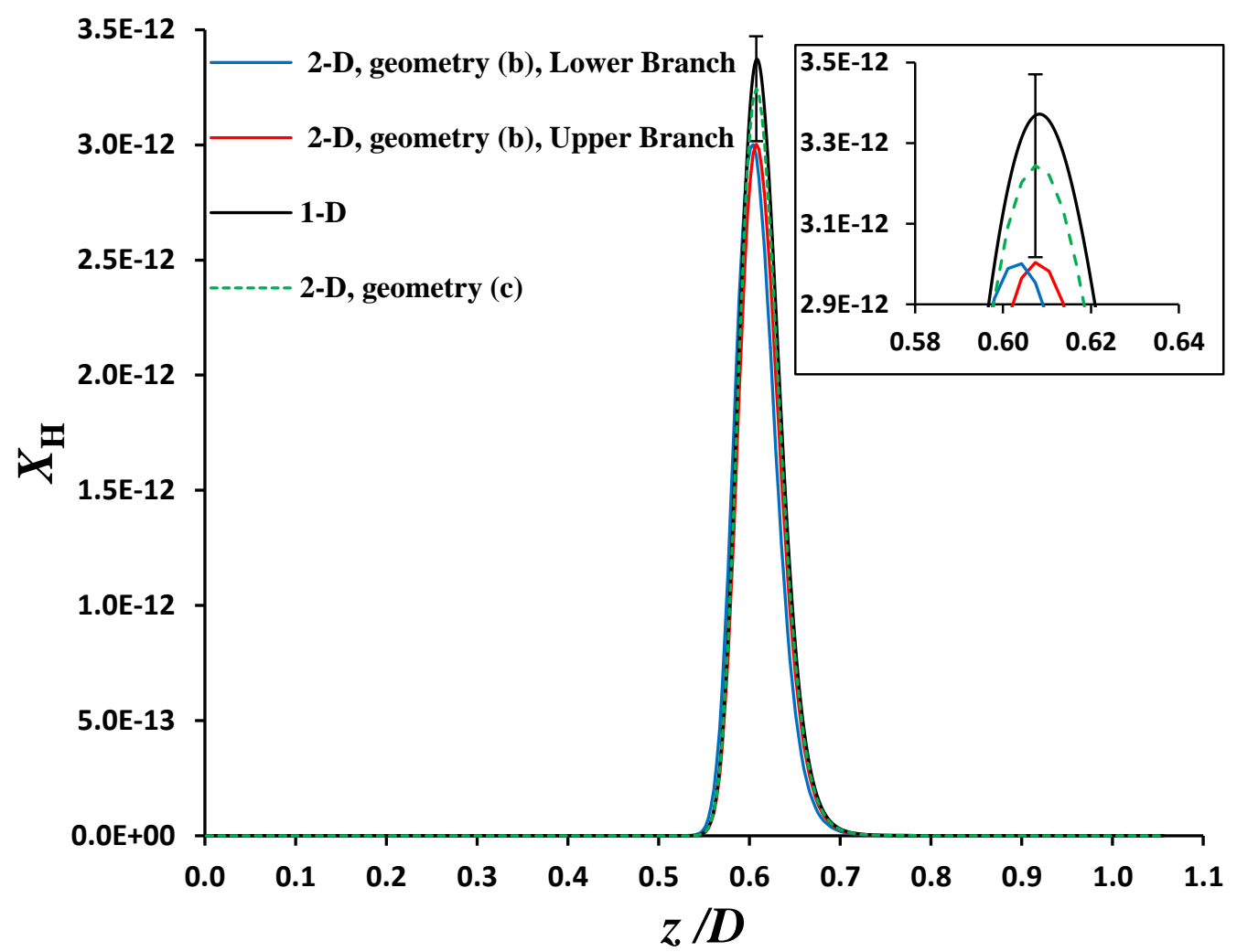

Fig. 17. Computed $\boldsymbol{X}_{\boldsymbol{H}}$ profiles along the centerline of geometry (b) and (c); inset: same profiles plotted close to the stagnation point to aid the comparison.

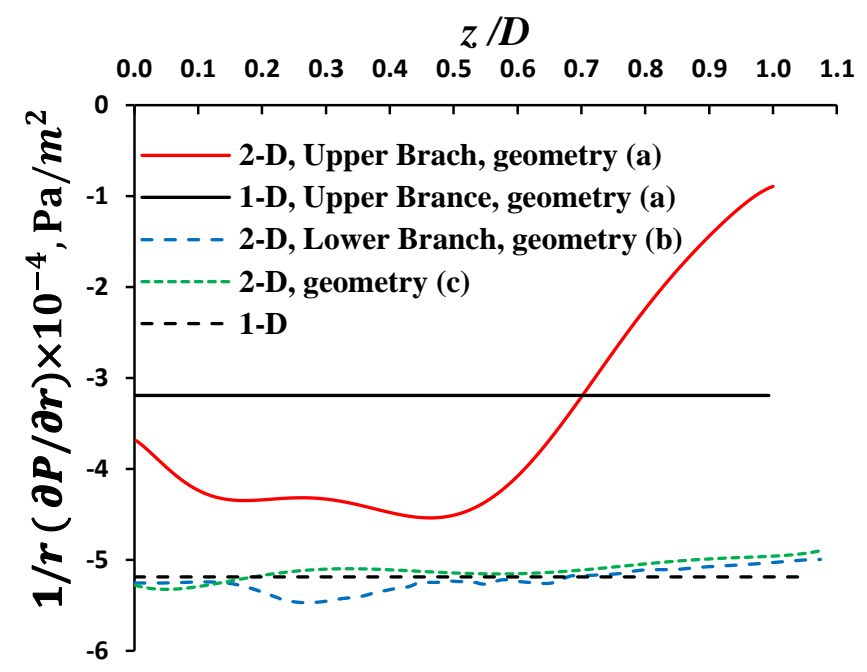

Fig. 18. Comparison between the typical computed axial profiles of radial pressure curvature along the stagnation streamline. 


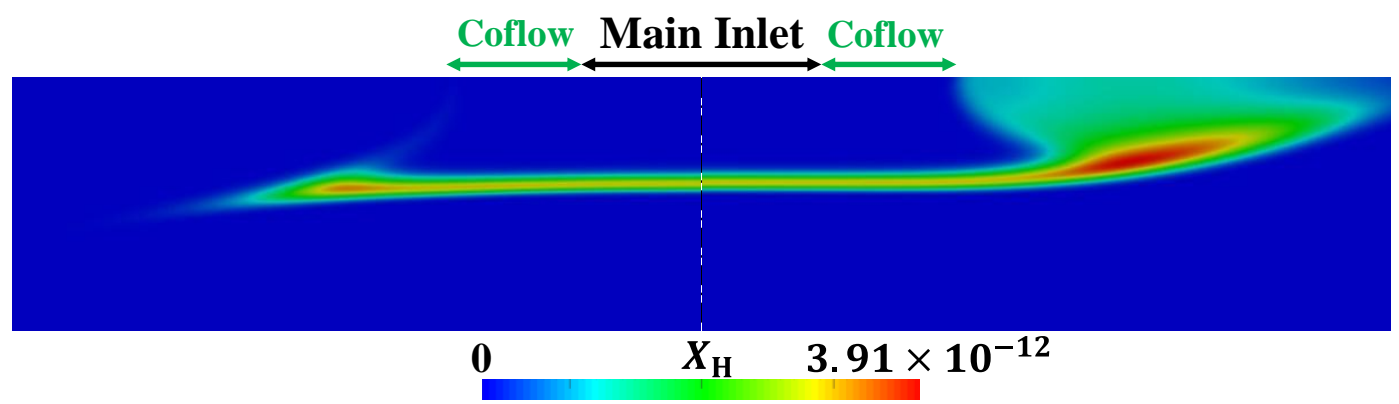

Fig. 19. Comparison between computed $\boldsymbol{X}_{\boldsymbol{H}}$ maps for two stable solutions of geometry (b); Left: lower branch; Right: upper branch.

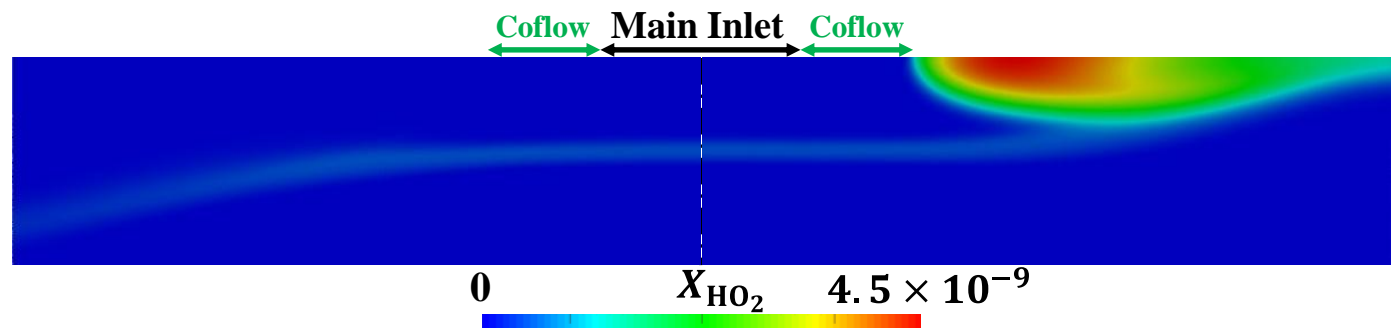

Fig. 20. Comparison between computed $\boldsymbol{X}_{\mathbf{H O}_{2}}$ maps for two stable solutions of geometry (b); Left: lower branch; Right: upper branch.

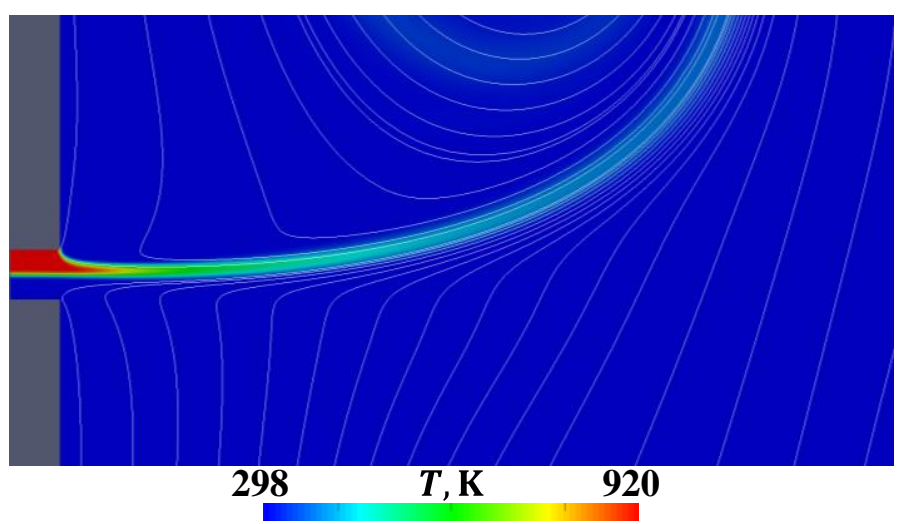

Fig. 21. Computed temperature map superimposed by the entrainment streamlines for the only stable solutions of geometry $(\mathrm{c})$. 


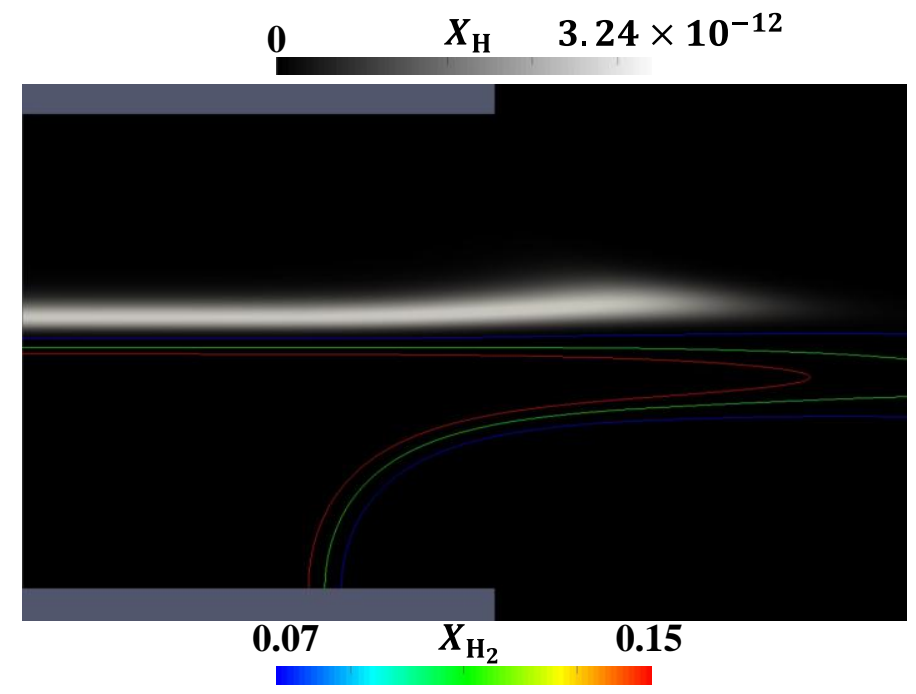

Fig. 22. Computed $\boldsymbol{X}_{\boldsymbol{H}}$ maps overlaid by $\boldsymbol{X}_{\mathbf{H}_{2}}$ iso-contours for geometry (c). 